\title{
Predicting spread and effective control measures for African swine fever-Should we blame the boars?
}

\author{
Rachel A. Taylor $^{1}$ (D) | Tomasz Podgórski ${ }^{2,3}$ (D) | Robin R. L. Simons ${ }^{1}$ (D) | Sophie Ip ${ }^{4}$ | \\ Paul Gale ${ }^{1}$ (D) | Louise A. Kelly ${ }^{1,5}$ (D) | Emma L. Snary ${ }^{1}$
}

${ }^{1}$ Department of Epidemiological Sciences, Animal and Plant Health Agency, Addlestone, UK

${ }^{2}$ Mammal Research Institute, Polish Academy of Sciences, Białowieża, Poland

${ }^{3}$ Department of Game Management and Wildlife Biology, Faculty of Forestry and Wood Sciences, Czech University of Life Sciences, Praha, Czech Republic

${ }^{4}$ Department of Applied Mathematics and Theoretical Physics, University of

Cambridge, Cambridge, UK

${ }^{5}$ Department of Mathematics and Statistics, University of Strathclyde, Glasgow, UK

\section{Correspondence}

Rachel A. Taylor, Department of Epidemiological Sciences, Animal and Plant Health Agency, Addlestone, UK.

Email:Rachel.taylor@apha.gov.uk

Funding information Horizon 2020 Framework Programme, Grant/Award Number: 643476; Narodowe Centrum Nauki, Grant/Award Number: 2014/15/B/NZ9/01933

\begin{abstract}
An ongoing, continually spreading, outbreak of African swine fever (ASF), following its identification in Georgia in 2007, has resulted in 17 European and 12 Asian countries reporting cases by April 2020, with cases occurring in both wild boar and domestic pigs. Curtailing further spread of ASF requires understanding of the transmission pathways of the disease. ASF is self-sustaining in the wild boar population, and they have been implicated as one of the main drivers of transmission within Europe. We developed a spatially explicit model to estimate the risk of infection with ASF in wild boar and pigs due to natural movement of wild boar that is applicable across the whole of Europe. We demonstrate the model by using it to predict the probability that early cases of ASF in Poland were caused by wild boar dispersion. The risk of infection in 2015 is computed due to wild boar cases in Poland in 2014, compared against reported cases in 2015, and then the procedure is repeated for 2015-2016. We find that long- and medium-distance spread of ASF (i.e. $>30 \mathrm{~km}$ ) is unlikely to have occurred due to wild boar dispersal, due in part to the generally short distances wild boar will travel ( $<20 \mathrm{~km}$ on average). We also predict the relative success of different control strategies in 2015, if they were implemented in 2014. Results suggest that hunting of wild boar reduces the number of new cases, but a larger region is at risk of ASF compared with no control measure. Alternatively, introducing wild boarproof fencing reduces the size of the region at risk in 2015 , but not the total number of cases. Overall, our model suggests wild boar movement is only responsible for local transmission of disease; thus, other pathways are more dominant in mediumand long-distance spread of the disease.
\end{abstract}

KEYWORDS

dispersal, fencing, hunting, intervention strategies, risk assessment, risk of infection, wild boar movement 


\section{1 | INTRODUCTION}

African swine fever (ASF) is a porcine disease that is rapidly spreading throughout Europe and Asia. Due to its high mortality rates and ability to spread within and between farms, preventative control measures including culling of animals are usually implemented (Costard, Mur, Lubroth, Sanchez-Vizcaino, \& Pfeiffer, 2013). ASF is notifiable to the World Organisation for Animal Health (OIE), which can lead to trade restrictions from other countries. National or international legislation may enforce additional control and surveillance measures to minimize the risk of international spread through trade, which could result in devastating economic impacts. Combined trade restrictions, the financial impact of the disease and costly control measures indicate the potentially devastating economic impacts that are possible. The disease is endemic in sub-Sahara Africa, where there is a domestic pig-to-pig cycle, a sylvatic cycle with warthogs, bush pigs and wild pigs and a tick-pig cycle (Penrith, Bastos, Etter, \& BeltránAlcrudo, 2019). However, an epidemic is currently ongoing in Asia and Europe, first identified in Georgia in 2007, quickly followed by cases in Russia, with Ukraine and Belarus also reporting cases in 2012 and 2013, respectively. In 2014, Lithuania and Poland were the first European Union (EU) countries to report ASF cases and since then there has been steady spread to neighbouring countries namely (as of April 2020) Latvia, Estonia, Moldova, Hungary, Romania, Bulgaria, Slovakia, Serbia and Greece, as well as jumps westward to the Czech Republic and Belgium (Cwynar, Stojkov, \& Wlazlak, 2019; OIE, 2020). Furthermore, in August 2018 the first reported cases of ASF in China occurred. By April 2020, there have been 165 outbreaks reported in 32 different provinces and spread to Mongolia, North Korea, South Korea, Lao, Myanmar, Vietnam, Cambodia, Indonesia, Papua New Guinea, Timor-Leste and the Philippines (OIE, 2020). Its arrival in China, with the world's largest pork industry, highlights the seriousness of this disease for the global pig industry. There are many transmission pathways that have been implicated in the recent epidemic in Europe and Asia (Chenais et al., 2019) although the role of ticks is inconclusive (Sánchez-Cordón, Montoya, Reis, \& Dixon, 2018). Due to the predominance in the European epidemic of pig cases being in areas where ASF-infected wild boar have also been found, especially in Latvia, Lithuania, Estonia and Poland, one of the major transmission pathways to pigs is considered to be contact with this wild boar population (Chenais et al., 2019). Natural movement of wild boar, including both home-range movement and long-range dispersal, could lead to contact of infected wild boar with both susceptible wild boar and pigs in uninfected areas, thus spreading the disease away from the initial source. However, if biosecurity measures to reduce contact between pigs and wild boar are implemented on farm, then a more probable transmission route to pigs may be human-mediated transmission, such as contaminated clothing and movement of animals. Illegal practices are also a concern; in China, the majority of the cases appear to be related to the process of swill feeding (FAO, 2019). Human transportation of infected meat is perhaps most likely to have led to the long-distance jumps of ASF (Chenais et al., 2019), such as to the Czech Republic and Belgium which were several hundred kilometres away from the nearest case (EFSA et al., 2018), although contaminated trucks and movement of infected pigs, wild boar or wild boar carcasses are also possible transmission routes (Chenais et al., 2019; EFSA et al., 2018).

We focus on the role of wild boar in order to determine the extent to which natural movement of wild boar could be responsible for the spread of ASF at different distances. To do this, we create a generic model for the risk of introduction of disease via wild animal terrestrial movement, that is appropriate for a broad range of species, and parameterize it for wild boar. Other models have also considered the role of wild boar movement in the transmission of ASF within Europe. For example, De la Torre et al. (2015) and Bosch et al. (2017) assess the risk of introduction of ASF due to wild boar at a country level for those countries free of ASF using a semi-quantitative method. Quantitative methods include those of Simons et al. (2019), which assess risk of entry at a country level across Europe, using wild boar abundance and suitability at a fine scale to compute this risk, and the models of Thulke and Lange (2017) and Lange, Guberti, and Thulke (2018). In the latter two, wild boar movement is modelled on a fine spatial scale using individual-based modelling but on an abstraction, that is the spread of disease is not based on an actual European map but on a representative square grid. Alternatively, Iglesias et al. (2018) and Podgórski and Śmietanka (2018) use statistics to assess the role of wild boar movement in driving the outbreaks of ASF at various locations in Russia and Poland, respectively. The former found that wild boar were responsible for $55 \%$ of new infections of ASF in their study area, whereas the latter found no significant correlation between statistics describing wild boar movement and new cases of ASF. In this paper, we take the wild boar movement model of Simons et al. (2019), but adapt it to be both more generic in its spatial scale and assessing infection rather than introduction risk, so that it fits within the aims of the COMPARE model framework (Taylor, Berriman, Gale, Kelly, \& Snary, 2019) (see below for further details on this framework). Thus, this model is based upon actual European data for wild boar abundance as well as prevalence of cases, in order to assess risk of infection for specific locations in Europe, using Poland as a case study. We do not use an individual-based model like Thulke and Lange (2017) because we require our model to be generic to other terrestrial wild animals and due to the complexity it would involve on a large spatial scale, if expanding the model to assess risk across the whole of Europe.

The abundance of wild boar in Europe has been increasing over recent decades alongside an expanded range within Europe and colonization of new habitats, including urban areas (Massei et al., 2015; Sáaez-Royuela \& Telleriia, 1986). This has prompted research on wild boar ecology, such as their movement, location and the suitability of habitat across Europe to sustain wild boar populations (Morelle et al., 2015). Wild boar exist in matrilineal groups, with separate home ranges for males and females and little interaction outside of the breeding period (D'Eath \& Turner, 2009; Podgórski, Scandura, \& Jędrzejewska, 2014). Males tend to have larger home ranges than females, with both varying seasonally. The main factor in long-distance movement of wild boar is the post-weaning of young males 
in search of new territory. This dispersal event usually involves a long-distance movement outside of the natal home range for at least $40 \%-50 \%$ of young male wild boar between 6 months and 2 years old (Podgórski et al., 2014; Truvé \& Lemel, 2003). Female wild boar may also choose to disperse, although usually for shorter distances than males (Podgórski et al., 2014). The combination of home range and dispersal is a significant factor in the difficulty in predicting the effects of the movement on disease spread by wild boar, especially since it is unclear how infection affects individual wild boar and their propensity to move longer distances. Although infected wild boar would still be able to disperse during their latent period, the virulence of the disease may reduce wild boar dispersal distance or the proportion of wild boar dispersing. As wild boar do not usually have as frequent or close monitoring as commercial pig holdings (OIE, 2015), infected animals may not be detected and removed as quickly. Hence, not only can infected wild boar continue to have contact with other wild boar and potentially domestic pigs while alive and infected, but also after death the carcass can remain in the environment for months (Probst, Globig, Knoll, Conraths, \& Depner, 2017). ASF virus is known to have a long survival time in the carcass and surrounding environment (Mebus et al., 1993) and studies have shown it is not uncommon for wild animals, including other wild boar, to forage around dead carcasses (Probst et al., 2017; Selva, Jędrzejewska, Jędrzejewski, \& Wajrak, 2005). Thus, transmission from carcasses is an important transmission route (Pepin, Golnar, Abdo, \& Podgorski, 2020) and we also consider its role in transmission within our model.

Many countries and agencies are eager to know how best to control, manage and hopefully eradicate ASF, in order to stop the continued spread throughout the world and the deleterious effects on the pig industry. A number of control strategies are implemented within the EU based on a EU directive (European Union, 2002) and working document (European Union, 2018), such as culling, cleansing and disinfecting on infected farms, testing and removal of any wild boar carcasses found and increased awareness and promotion of biosecurity measures. These documents also involve creating different zones around the infected cases-an infected and a surveillance zone-with different controls implemented. Additional measures, such as fencing, are left up to the relevant country. For example, in both Belgium and the Czech Republic after the first cases were discovered, fences were built surrounding the infected zone and no hunting or feeding of wild boar was allowed within the area. Furthermore, all human activity in the area, including hunting, was stopped or scaled back to reduce both potential transmission from, for example, contaminated clothing, and the disturbance it causes in the wild boar population, which can lead to increased wild boar movements. However, in the wider surveillance zone, hunting occurred to reduce the wild boar population outside the infected zone. In both zones, increased passive surveillance was undertaken aiming to determine the extent of the disease and reduce the length of time wild boar carcasses may be in the environment. In the Czech Republic and Belgium, this has been successful (so far) in preventing further local spread of the disease (Mlynar, 2018; PAFF Committee, 2019).
In this manuscript, we describe the development of a model of wild boar movement that is valid across Europe. This model is not specific to ASF, other than through the choice of disease-related parameter values, and thus, it can also be used to model the spread of other infectious diseases via wild boar movement, such as classical swine fever, foot and mouth disease and Aujeszky's disease. In fact, we choose instead to create a generic model of wild animal movement, by focussing on those aspects of the model which are similar across many terrestrial species, such as home-range and long-range dispersal and group structure, rather than building a bespoke wild boar model. For example, the framework could be used to model the spread of rabies via foxes. The reason to make the model generic is to ensure the possibility of rapidly modelling disease spread within disease outbreaks for many diseases and species, which is crucial if models are to inform on surveillance, intervention and disease progression during an outbreak (Miller \& Pepin, 2019).

We apply the wild animal movement model to a case study of the early cases of ASF in Poland. These initial Polish cases in 2014 were all in wild boar, localized and in close proximity to the border with Belarus, where 2 cases had been reported in 2013, suggesting transboundary introduction as the initial source (Pejsak, Truszczyński, Kozak, \& Markowska-Daniel, 2014). We consider whether wild boar movement alone can be responsible for the cases that occurred in 2015 and 2016 by predicting the probability of at least one infection in $2015 / 2016$ based upon the location of cases in $2014 / 2015$. We then compare our predicted probability of at least one infection in $2015 / 2016$ against the locations of reported cases in 2015/2016. We also use the model to assess the effectiveness of three control strategies, (a) increasing the removal rate of wild boar carcasses, (b) hunting and (c) fencing, by comparing against the baseline predicted risk in 2015 without the control strategies. The aim was to understand in detail how transmission of ASF due to wild boar movement can occur in order to determine more accurately the risk of infection of ASF across Europe.

\section{2 | METHODS}

\section{1 | Overview of the risk assessment model}

We model the risk of infection with ASF virus within new areas in Europe due to wild boar movement using the spatial quantitative risk assessment framework outlined in Taylor et al. (2019). This framework incorporates many disease entry pathways and for each considers the number of infectious animals (or infectious units depending on the entry pathway) entering a new Area B given that the disease is present in Area A, whether detection of these animals would occur, survival of these animals in the new Area $B$, and contact and transmission with susceptible animals in Area B. Adopting this framework, we calculate the risk of initial infection with ASF virus using a cell structure across Europe where each cell is $100 \mathrm{~km}^{2}$, that is with sides of $10 \mathrm{~km}$. Area $A$ is defined as those cells which we estimate to have non-zero incidence (see the section on incidence 
below for how we calculate this). Area B is any cell within Europe (regardless of whether cases have already been reported in that cell). Following Taylor et al. (2019), the number of infected wild boar reaching cell $c$ of Area $B$ is given by:

$$
I_{k}(c) \sim \operatorname{Bin}\left(N_{k}(c), p_{k}\right),
$$

where $k$ represents a cell in Area $A, N_{k}(c)$ is the number of wild boar moving from cell $k$ to cell $c$ and $p_{k}$ is the prevalence in cell $k$. We thus define $I(c)=\sum_{k} I_{k}(c)$ as the total number of infected wild boar entering cell $c$ from any cell $k$. We assume no detection of infection in wild boar will occur during their movement from one cell to another.

We model the contact with susceptible wild boars and pigs using the disease metric $R_{0}$, which is a measure of the expected number of infections that would occur if one infected wild boar were to enter a susceptible population. Our equation for $R_{0}(c)$ includes information on the susceptible populations at risk in cell $c$, the pathway of entry, the transmission between infected and susceptible animals and the survival of infection. Each parameter from $R_{0}(\mathrm{c})$ is drawn from a distribution of likely values. The number of initial infections, $N_{1}(c)$, in cell $c$ is then calculated by $I(c)$ draws from a Poisson distribution with mean given by $R_{0}(\mathrm{c})$ :

$$
N_{l}(c)=\sum_{1}^{I(c)} \text { Pois }\left(R_{0}(c)\right)
$$

That is, there are $I(c)$ infected wild boar entering cell $c$, each of which has a mean probability of infecting $R_{0}(c)$ susceptible animals, and the Poisson distribution outputs a non-negative integer as to how many are actually infected. By summing this over all the infected wild boar entering, we get $N_{1}$ (c) new infections. The probability, $R_{1}(\mathrm{c})$, that at least one infection in a susceptible animal would occur in cell $c$ is then given by the proportion of the simulations where infection occurs in a susceptible wild boar or pig. We outline in greater detail below how we compute each step of the risk assessment for the specific wild boar movement pathway.

\subsection{The wild boar movement model}

We model the number of wild boar moving from Area $A$ to Area $B$ based on wild boar movement ecology, using the cell structure mentioned, on a yearly timescale. We adapt a model of Simons et al. (2019), which models the number of infected wild boar which will reach the border of a new country. However, we enhance the model to estimate at a cell level where the wild boar are likely to travel to, regardless of country borders. Due to the desire for the movement model to be applicable for large spatial extents, such as the whole of Europe, and the computational resources required to do so, the calculation of movement of wild boar is deterministic. For each cell in Area A, we estimate where those wild boar will be after a year, depending on whether they perform one or two types of movement, namely movement within their home-range (hereafter referred to as home-range movement) and long-range dispersal. Long-range dispersal may be undertaken by adult male and female wild boar but is predominantly undertaken by young male adults. To represent this in the model, a proportion of all wild boar undergo long-range dispersal. For long-distance movement, we assume the direction the wild boar moves is determined by the habitat suitability of each cell, with wild boar moving to neighbouring cells of highest habitat suitability, where habitat suitability is a measure of how suited each cell is for wild boar to live and is given by a score between 0 and 1 (Alexander, Massei, \& Wint, 2016). Other factors could also influence where wild boar move to, such as competition for resources, but we do not include these. Thus, wild boar long-range movement is a biased random walk, characterized by the benefit they will receive from moving to the neighbouring cells, to represent the fact that wild boar are searching for better quality habitats when dispersing (Truvé \& Lemel, 2003). Given the long-range distance that wild boar travel, we fix a total number of steps (defined as a movement from one cell to a neighbouring cell) the wild boar can travel, $n$, to be the total distance in $\mathrm{km}$ divided by the width of a cell, in our case $10 \mathrm{~km}$. We use $N(c)$ to indicate the neighbourhood of each cell $c$, specifically the 4 cells to the north, east, south and west, the 4 corners, and the cell itself, and we usec̃ to represent a neighbouring cell of cell $c$. Thus, wild boar can choose to stay in their current cell if it has a high suitability. The probability that a wild boar will be in cell $c$ after $n$ steps, $p(c, k, n)$, given that the wild boar started from cell $k$ in Area $A$, is then given by the recurrence relation:

$$
p(c, k, n)=\sum_{\tilde{c} \in N(c)} B(c, \tilde{c}) p(\tilde{c}, k, n-1),
$$

where $B(c, \tilde{c})$ is a probability of moving from cell $\tilde{c}$ to cell $c$ determined by the benefit a wild boar will receive by moving, and is multiplied by the probability of being in cell $\tilde{c}$ after $n-1$ steps. The recurrence relation has initial conditions at step 0 of:

$$
\left\{\begin{array}{l}
p(c, k, 0)=1 \text { if } c=k \\
p(c, k, 0)=0 \text { if } c \neq k
\end{array}\right.
$$

Note that for cells neighbouring $k$, the origin cell, we do not allow movement back to $k$ in order to ensure dispersal from the home range does actually take place. The benefit probability $B(c, \tilde{c})$ is based on the habitat suitability score, $h(c)$, in each cell $c$. It is calculated by comparing the difference in the suitability between the two cells $c$ and $\tilde{c}$ and normalizing this by the benefit that could be gained by moving to any of the neighbouring cells $c^{*}$ of cell $\tilde{c}$ :

$$
B(c, \tilde{c})=\frac{1+h(c)-h(\tilde{c})}{X_{c^{*} \in N(\tilde{c})}\left(1+h\left(c^{*}\right)-h(\tilde{c})\right)}
$$

Since most wild boar dispersal is by young adult wild boar which perform the long-range movement in search of new territory usually only once in their lifetime, we model one movement event of wild 
boar and then assess the risk for a year, but do not specifically model the time that events occur within the year. Once wild boar arrive at their final destination, we assume they perform home-range movement in this new cell for the rest of the year. We multiply $p(c, k, n)$, the probability that a wild boar from cell $k$ is in cell $c$ after $n$ steps, by the number of wild boar in cell $k$, and by a probability, $p_{i}$, that the wild boar will be infected at the same time as their long-distance dispersal. This probability, $p_{i}$, is derived from the latent period in days for indirect contact divided by 365 (Table 1). For home-range movement, we assume that the wild boar can travel anywhere within the home-range area regardless of the habitat suitability and have equal likelihood to contact all other wild boar in the home range (although see below for specifics about within- and between-group contact). In practice, the home range is smaller than the cell size, and therefore, we assume that the wild boar can explore a percentage of the cell during home-range movement, dependent on the home-range size. Therefore, if $S(c)$ is the number of susceptible wild boar in cell $c$, a wild boar performing home-range movement could have contact with $p_{s} S(c)$ susceptible wild boar. Here, $p_{s}$ is the proportional size of the home range compared with the cell size. This means that wild boar that undergo home-range movement can only infect susceptible wild boar which are in their origin cell, as they will not move to other cells. The final number of wild boar moving from Area $A$ to Area $B$, calculated from summing the number to each cell via homerange and long-distance dispersal, is then input into eqn 1 as $N_{k}(c)$.

\subsection{Abundance and suitability maps}

We use wild boar abundance and habitat suitability maps from Alexander et al. (2016) although alternative wild boar abundance maps have been created (e.g. Lewis et al. (2017)). To create the habitat suitability map, Alexander and co-authors used land cover databases, published descriptions of boar preferences and expert opinions. Combining the habitat suitability map with abundance-related data, from sources such as national and international databases and hunting records, within a species distribution model, they produced a European wide estimate of boar abundance. They outputted the wild boar abundance estimates on a semi-quantitative scale of 0-4 for each cell, rather than as specific values. We reconstructed

TABLE 1 Parameter values with a description and reference. Rates and times are given in units of years unless specified otherwise

\begin{tabular}{|c|c|c|}
\hline Parameter description & Value & Reference \\
\hline $\begin{array}{l}\text { Proportion of cell explored during home-range } \\
\text { movement }\left(p_{s}\right)\end{array}$ & 0.05 & $\begin{array}{l}\text { Based on Massei et al. (1997), Leaper } \\
\text { et al. (1999), Podgórski et al. (2014) }\end{array}$ \\
\hline Number of steps during long-range movement $(n)$ & 5 & Truvé and Lemel (2003) \\
\hline $\begin{array}{l}\text { Proportion of wild boar performing long-range } \\
\text { movement }\end{array}$ & $15.4 \%$ & Keuling et al. (2010) \\
\hline Infectious period in live wild boar (1/r) & $\begin{array}{l}\text { Pert }(\min =3 / 365, \text { mode }=6 / 365 \\
\max =10 / 365)\end{array}$ & $\begin{array}{l}\text { Based on Gabriel et al. (2011), Guinat } \\
\text { et al. (2014) }\end{array}$ \\
\hline $\begin{array}{l}\text { Probability of being infectious during long-range } \\
\text { movement }\left(p_{i}\right)\end{array}$ & $8 / 365$ & Guinat et al. (2014) \\
\hline Group size of wild boar (G) & 7 & Podgórski et al. (2014) \\
\hline $\begin{array}{l}\text { Per capita contact rate between wild boar and pigs in } \\
100-\mathrm{km}^{2} \text { cell with live wild boar }\left(\gamma_{W}, \gamma_{B}, \gamma\right)\end{array}$ & $\begin{array}{l}\text { Within wild boar group } \gamma_{W} \text { : } \\
\text { norm }(0.59,0.02)^{*} 365 \\
\text { Between wild boar groups } \gamma_{B} \text { : } \\
\text { norm }(0.035,0.002)^{*} 365 \\
\text { Wild boar to pig } \gamma \text { : unif }(0,0.267)\end{array}$ & $\begin{array}{l}\text { Podgórski et al. (2018) } \\
\text { Kukielka et al. (2013) }\end{array}$ \\
\hline Probability of transmission $(\beta)$ & $\begin{array}{l}\text { Wild boar to pig: unif }(0,0.167) \\
\text { Wild boar to wild boar: } \operatorname{pert}(0,0.167 \text {, } \\
0.3) \\
\text { Dead wild boar to wild boar: unif( } 0 \text {, } \\
0.167)\end{array}$ & $\begin{array}{l}\text { Estimated from Pietschmann et al. (2015) and } \\
\text { assumption }\end{array}$ \\
\hline Probability of lethal infection in wild boar $\left(p_{L}\right)$ & unif $(0.95,1)$ & $\begin{array}{l}\text { Blome, Gabriel, Dietze, Breithaupt, and Beer } \\
\text { (2012), Thulke and Lange (2017) }\end{array}$ \\
\hline $\begin{array}{l}\text { Probability that a dead wild boar will have direct } \\
\text { contact with live wild boar }\left(p_{d}\right)\end{array}$ & 0.5 & Probst et al. (2017) \\
\hline Yearly direct contact rate with wild boar carcass $\left(p_{d}\right)$ & $1 / 370 * \operatorname{norm}(179.5,73)$ & Probst et al. (2017) \\
\hline Length of infectious period for wild boar carcasses $\left(T_{s}\right)$ & $\operatorname{pert}(15 / 365,26 / 365,124 / 365)$ & $\begin{array}{l}\text { Estimated from Morley (1993), Probst } \\
\text { et al. (2017), Olesen et al. (2018), Chenais } \\
\text { et al. (2019) }\end{array}$ \\
\hline Probability that a carcass will be removed & $1 / 4$ & Estimated from the under-reporting factor \\
\hline Under-reporting factor & 4 & Adapted from Adkin et al. (2004) \\
\hline Number of simulations & 10,000 & \\
\hline
\end{tabular}


a numerical value for wild boar density per cell by manually curating the papers cited by Alexander et al. (2016), and extracting the data values from these papers to calculate population densities (see Appendix A). We then calculated the quartiles of our population estimates so that we could substitute the semi-quantitative scores of Alexander et al. (2016) with the quartile values instead. For both maps, Alexander et al. (2016) produce output on a $1-\mathrm{km}^{2}$ resolution. However, we aggregate the cell resolution up to $100 \mathrm{~km}^{2}$ to speed up calculations within the movement model.

Our model calculates the probability of initial infection of ASF occurring in a susceptible population, whether that population is wild boar or pigs. Therefore, we need abundance maps of the susceptible animal populations as well. For the wild boar population, we use the same wild boar abundance maps as above, but we reduce the number of animals in each cell by the number of estimated cases of ASF in wild boar in that cell in order to get a total number of susceptible wild boar. For the pig density maps, we use data from the FAO-gridded livestock of the world (FAO, 2014).

\subsection{Incidence in wild boar}

We estimate the total number of wild boar infected with ASF virus in each $100-\mathrm{km}^{2}$ cell in Poland over the year by extracting data on the number of cases of ASF from Empres-i, including case locations. We use yearly incidence rather than prevalence so that we can subsequently calculate whether the infected wild boar will be infectious at the time of their dispersal or home-range movement. Empres-i gathers its information on outbreaks from multiple locations, although the major source is the OIE, a comprehensive source due to the requirement for countries to report notifiable disease occurrence to the OIE. Other sources include FAO officers, European Commission and media. We aggregate the total number of wild boar cases by each $100-\mathrm{km}^{2}$ cell and then multiply this total by an under-reporting factor (see Table 1 for parameter values). Since all wild boar hunted or found dead in Poland are tested for ASF, this under-reporting factor is predominantly comprised of the likelihood of finding carcasses. We then calculate a first estimate of the incidence by dividing the inflated (by under-reporting) number of cases by the number of wild boar in that cell. However, it is possible that cases have occurred in neighbouring cells and also have not been found, correctly diagnosed or reported, since wild boar move irrespective of our cell boundaries. We therefore perform a smoothing method to the incidence data to account for this, which spreads our inflated cases across neighbouring cells. It does this by recalculating the incidence in all cells as a weighted average between the current incidence in that cell and the incidence in all 8 neighbours, with $50 \%$ weight given to the current cell and 50\% weight distributed equally among the neighbouring cells. We then recalculate the estimated number of cases in each cell by multiplying the number of wild boar by the new incidence estimate. Smoothing the incidence and then recalculating the number of cases (rather than smoothing the number of cases) ensure that we take into account the abundance of wild boar and hence the fact that some cells may have no cases due to no wild boar being present in that cell rather than under-reporting. Estimated incidence based on the cases in Poland in 2014 is plotted in Figure 1.

\subsection{Contact and transmission}

We create two separate equations for $R_{0}(c)$ in order to separately assess contact and transmission with live pigs and with live wild boar. We model the contact with susceptible pigs within each cell $c$ by considering how many susceptible animals are in the cell $(S(c))$, the length of the infectious period $(1 / r)$ and the per capita contact rate $(\gamma)$ and transmission probability $(\beta)$ between species:

$$
R_{0}(c)=\frac{\beta \gamma p_{S} S(c)}{r}
$$

We multiply $S(c)$ by $p_{s}$, as stated earlier, because wild boar will not roam the whole cell but only a proportion of it.

Wild boar predominantly exist in matrilineal groups (Podgórski et al., 2014). Therefore, for wild boar contact with other live wild boar we use the equation as above but modify it to include both within-group contact and between-group contact, by replacing $\gamma p_{s} S(c)$ with

$$
\gamma_{W} G+\gamma_{B}\left(p_{S} S(c)-G\right)
$$

In this equation, $\gamma_{w}$ is the within-group contact rate, $G$ is the average group size and $\gamma_{B}$ is the between-group contact rate which is applicable for contact with all other wild boar in the home range. We assume that only a proportion of wild boar survive infection, given by a probability that ASF infection is lethal in wild boar, $p_{L}$ (see Table 1). The carcasses of wild boar that died due to ASF can also contribute to transmission of ASF virus. For these carcasses, we estimate how many live wild boar will have direct contact with

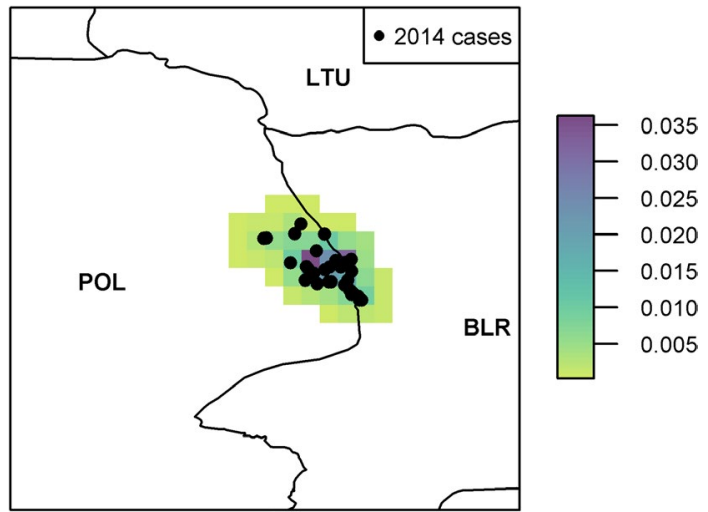

FIGURE 1 The estimated incidence of ASF at a $100-\mathrm{km}^{2}$ cell level, given the reported cases in Poland in 2014 and the wild boar abundance. Incidence is represented at a cell level by the colour scale on the right. Black circles indicate the locations of reported cases of ASF (which may have had $>1$ infected wild boar). White indicates zero incidence. Countries are indicated by their ISO3 code 
the carcass and thus able to become infectious by using a study of Probst et al. (2017), in which data on live wild boar contact with wild boar carcasses are collected. We compute the number of new cases of ASF due to carcasses as follows:

$$
\frac{p_{d} \gamma_{d} \beta_{c} S(c)}{r_{c}}
$$

Here, $p_{d}$ is the probability that direct contact will occur with a carcass (direct contact does not always occur due to the time of the year of death, location of carcass, etc.), $\gamma_{d}$ is the total number of direct contacts per year each wild boar has with a carcass, $\beta_{c}$ is the transmission probability from a carcass to a susceptible animal per contact, and $r_{c}$ is the rate at which the carcass is available to cause infection, which is the inverse of the length of time the carcass is available $\left(T_{c}\right)$. This is determined by two factors, skeletonization of the carcass and whether the carcass is removed, as follows:

$$
r_{c}=\frac{1}{T_{c}}=\frac{1}{\left(1-p_{r}\right) T_{S}+p_{r} T_{r}}
$$

where $T_{S}$ is the time until skeletonization of the carcass, $T_{r}$ is the time until removal of a carcass and $p_{r}$ is the probability that a wild boar carcass is found and removed.

Therefore, our full equation for $R_{0}(c)$ in susceptible boar populations, that is, the likelihood of new cases occurring in susceptible wild boar in cell $c$ given an infected wild boar has entered the cell, is as follows:

$$
R_{0}(c)=\frac{\beta\left(\gamma_{W} G+\gamma_{B}\left(p_{S} S(c)-G\right)\right)}{r}+p_{L}\left(\frac{p_{d} \gamma_{d} \beta_{c}}{r_{c}}\right) S(c) .
$$

We then calculate the number of new infections in wild boar or pigs occurring in cell $c$ because of infected animals $I(c)$ moving in to cell $c$ by eqn 2 . For our results, firstly we calculate quantiles of the number of new infections in wild boar or pigs in each cell. Secondly, the overall probability of at least one infection in each cell is calculated based upon the proportion of simulations in which infection occurs in that cell. Lastly, we define the risk region as all of the cells which have non-zero probability of at least one infection.

\section{6 | Parameter values}

A full list of all parameter values, their descriptions and references is provided in Table 1. We outline the choice for key parameters. The parameters within the calculation of $R_{0}$ are represented by distributions in order to include variability in those rates and probabilities.

In order to calculate the proportion of the cell explored during home-range movement $\left(p_{s}\right)$, we used an average home-range size of $5 \mathrm{~km}^{2}$ based upon various studies (Leaper, Massei, Gorman, \& Aspinall, 1999; Massei, Genov, Staines, \& Gorman, 1997; Podgórski et al., 2014). For long-distance movement, the model considers how many steps wild boar will take based on how many kilometres they travel. Therefore, the parameter is defined as total distance travelled by wild boar rather than the straight-line distance from their starting point. Estimates for dispersal distance of wild boar are wide-ranging, such as $<30 \mathrm{~km}$ for most wild boar but up to $100 \mathrm{~km}$ possible (Truvé \& Lemel, 2003), a maximum dispersal distance of $41.53 \mathrm{~km}$ (Keuling, Lauterbach, Stier, \& Roth, 2010), 48 km (Lange, 2015) and even up to $250 \mathrm{~km}$ (Andrzejewski \& Jezierski, 1978). However, these estimates are all based on straight-line distances from original location. Therefore, we choose $50 \mathrm{~km}$ as the dispersal distance as this allows for longer movement, but most wild boar will disperse $<50 \mathrm{~km}$ from their original location similar to Truvé and Lemel (2003). Furthermore, it incorporates the fact that a wild boar with infection of ASF is unlikely to perform a very long movement (Podgórski \& Smietanka, 2018). Thus, the number of steps a wild boar can take, $n$, is 5 .

We calculate the contact rates between live wild boar and other live wild boar using Podgórski, Apollonio, and Keuling (2018) which estimated within-group and between-group contacts rates to be $0.59 \pm 0.02$ and $0.035 \pm 0.02$ per day, respectively. For wild boar contact with domestic pigs, we use a study which assessed direct contacts between wild boar and domestic pigs in central Spain on a 'free-roaming' farm (Kukielka et al., 2013). We divide the number of direct contacts over a year (8) by the total number of pigs (5) and wild boar (10) in the area, to produce 0.16 contacts per wild boar per pig in a year. The Spanish farm was $3 \mathrm{~km}^{2}$, whereas our home range is $5 \mathrm{~km}^{2}$ and so we convert the contact rate to our home-range size by multiplying by $5 / 3$ to reach a rate of 0.267 contacts per wild boar per pig. Since the domestic pigs were free-ranging on an open farm with no barriers, we use this as an upper limit in a uniform distribution, with a lower limit of 0 , to represent the fact that contact may not occur at all in a year depending on biosecurity of farms.

For contact between live wild boars and wild boar carcasses, we primarily used the study of Probst et al. (2017), which used cameras to assess the level of contact between live wild boar and wild boar carcasses over the course of a year. Of 32 carcasses laid out, 16 experienced direct contact (thus $p_{d}=0.5$ ). Of those 16 carcasses, 189 direct contact visits by live wild boar were counted, an average of 11.8 visits per carcass. We assume there is a normal distribution around this estimate with a standard deviation of 4.8 around 11.8 based on the average number of visits per carcass site. Due to skeletonization of the carcass, each carcass was approximately available for direct contact for 3.8 weeks (27 days) only. However, wild boar are unlikely to contact a wild boar carcass within the first 15 days (Probst et al., 2017), and therefore, the time period for potential wild boar contact with a carcass before skeletonization is 12 days. Therefore, our yearly contact rate (shown using the mean of carcass visits, 11.8) is $\gamma_{d}=11.8 \times\left(\frac{365}{12}\right)$, where $\frac{365}{12}$ converts the contact rate from a rate per 12 days to a rate per year. However, we convert this into a per wild boar contact rate by estimating the mean number of wild boar that were in the area where the carcasses were placed, in order to give a measure of how many wild boar could have contacted the carcass. To 
do this, we determine the $100-\mathrm{km}^{2}$ cell each carcass was placed in, use the wild boar abundance map to find out how many wild boar are estimated to be in that $100-\mathrm{km}^{2}$ cell, and then average across all carcasses. This gives an estimate of 370 wild boar that on average could have contacted the carcasses. Therefore, the contact rate per wild boar is calculated by dividing $\gamma_{d}$ by 370 .

The length of the infectious period for carcasses $\left(T_{c}\right)$ is also determined by the time until skeletonization $\left(T_{s}\right)$. As ASF virus has been found to survive in meat for up to 230 days (Morley, 1993), we assume that an infected carcass will be infectious until skeletonization is complete. However, we adjust the length of the infectious period to incorporate two factors-possible transmission after skeletonization through contact with both the soil surrounding the remaining bones and chewing the bones themselves, and the fact already mentioned that wild boar are unlikely to contact a carcass within the first 15 days (Probst et al., 2017). As above, we set 12 days to represent the average time before skeletonization during which wild boar may contact the carcass. Regarding the length of time in which wild boar may contact the remaining bones or soil below the carcass, there is no consensus over how long the virus could remain in the environment or bones. In one study, environmental transmission due to shedding of virus from infected live wild boar was found to be $<3$ days (Olesen et al., 2018). Chenais et al. (2019) state that ASF has been found experimentally to remain infectious in forest soil for 112 days. Further, Chenais et al. (2019) mentioned an unpublished study in which soil samples from carcass locations were PCR-positive 'several days or weeks after the carcasses has been removed although no viable virus could be isolated'. We therefore use a pert distribution of pert $(3,14,112)$ days to describe the length of time in which transmission could be possible after skeletonization.

We also include the probability of a wild boar carcass being found and removed $\left(p_{r}\right)$ and the length of time until this occurs $\left(T_{r}\right)$, to complete the calculation of $T_{c}$. For the probability of the wild boar carcass being found, we assume that all reported wild boar cases were removed, and hence, we use the inverse of the under-reporting factor in number of cases to estimate what proportion of the true cases are found and removed. The length of time until carcass removal is given by a uniform distribution from 1 day until the length of time until carcass skeletonization.

For the probability of transmission for a direct contact between a live wild boar and a carcass, we use a uniform distribution from 0 to a maximum of 0.167 due to a study by Pietschmann et al. (2015) in which wild boar are infected with a low dose of ASF corresponding to 'those obtained through contact with fomites, swill, excretions of infected animals, or contact with carcasses'. Of the 12 wild boar that were inoculated, 2 became infected, although those two were described as 'runted' prior to study commencement. For transmission between live wild boar and other wild boar, there are no studies estimating the transmission probability, and therefore, we assume a pert distribution with minimum of 0 and mode 0.167 (the value from carcass transmission), and for the maximum, we assume a value of 0.3 . The probability of transmission between live wild boar and pigs is lower than between wild boar (Pietschmann et al., 2015), and therefore, we assume the same distribution as for wild boar carcasses.

\section{7 | Scenario analyses}

We perform different scenario analyses to address the potential control strategies that could have been implemented in Poland to assess their effectiveness when wild boar alone are responsible for transmission. We assume that the control strategy is implemented in 2014 and compute a new risk for 2015 including the potentially positive and negative effects of the control strategy. We then compare our new results against the baseline results for 2015 .

The scenario analyses we perform are as follows:

A Increase in carcass removal (CR): This affects the probability of a carcass being found and removed; in the baseline model, 1 in 4 carcasses are removed

CR1. 1 in 3 carcasses are found and removed

CR2. 1 in 2 carcasses are found and removed

B Hunting $(\mathrm{H})$ : This affects both the number of wild boar in the area and the probability that wild boar will move due to disturbance $\mathrm{H} 1$. Reduction in wild boar population to half original size and $25 \%$ of remaining wild boars disperse

$\mathrm{H} 2$. Reduction in wild boar population to quarter of original size and $25 \%$ of remaining wild boars disperse

H3. Reduction in wild boar population to half original size and $50 \%$ of remaining wild boars disperse

H4. Reduction in wild boar population to quarter of original size and $50 \%$ of remaining wild boars disperse

C Fencing $(F)$ : The implementation of fencing around the current cases is determined by a buffer width and the permeability of the fence in successfully stopping wild boar moving outside the buffer

F1. Fence of $10-\mathrm{km}$ buffer and $95 \%$ successful

F2. Fence of $10-\mathrm{km}$ buffer and $50 \%$ successful

F3. Fence of $20-\mathrm{km}$ buffer and $95 \%$ successful

F4. Fence of $20-\mathrm{km}$ buffer and $50 \%$ successful

See Appendix B for an explanation of how the scenario analysis was implemented in the model. The parameter values chosen for the scenarios, such as permeability of the fence or increased dispersal, were chosen to represent potential scenarios rather than specific control strategies, although the increase in dispersal is based upon Thurfjell, Spong, and Ericsson (2013) in which 6 out of 15 radio-tracked female wild boar dispersed due to hunting disturbance.

We assess the effectiveness of these 10 control scenarios by comparing them against the baseline scenario using three metrics assessing severity and spread, namely the total number of cases per simulation; the total number of cells which have cases per simulation; and the total number of cells which have a non-zero probability of at least 1 case over the 10,000 simulations. Thus, the first two 


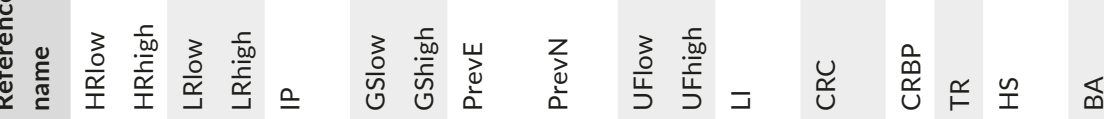

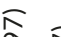

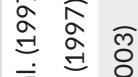

范

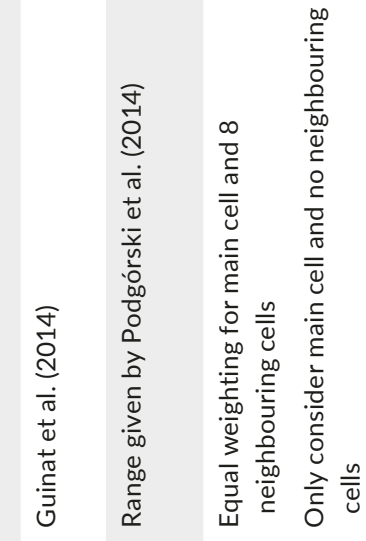

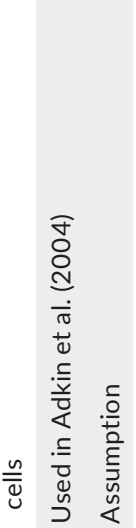

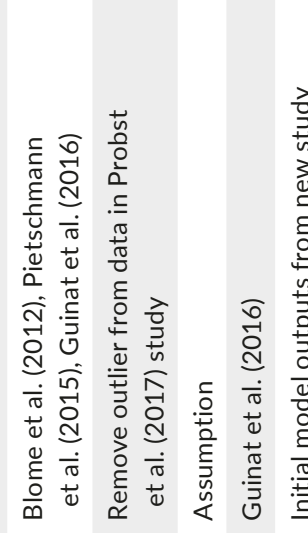

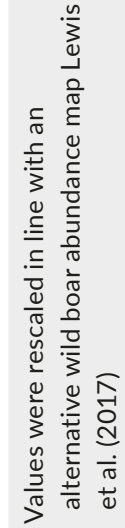

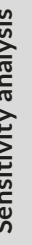

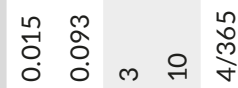

$\stackrel{\substack{q \\ m}}{n}$

ล คำ

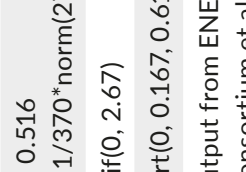

苛
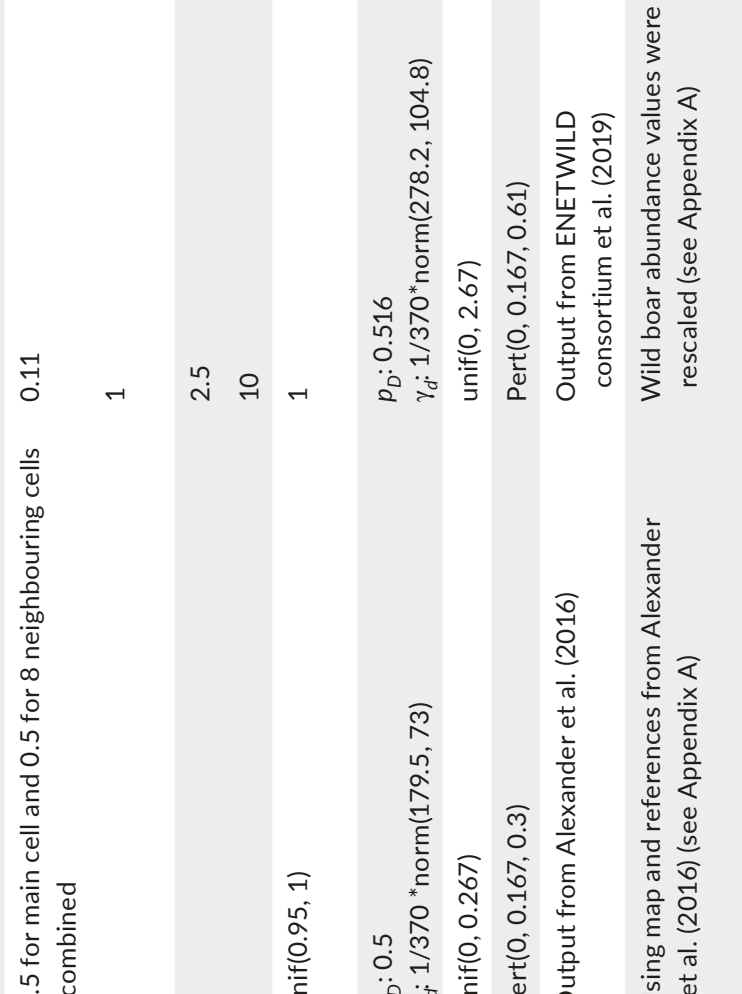

$\stackrel{n}{0}$
$\infty$
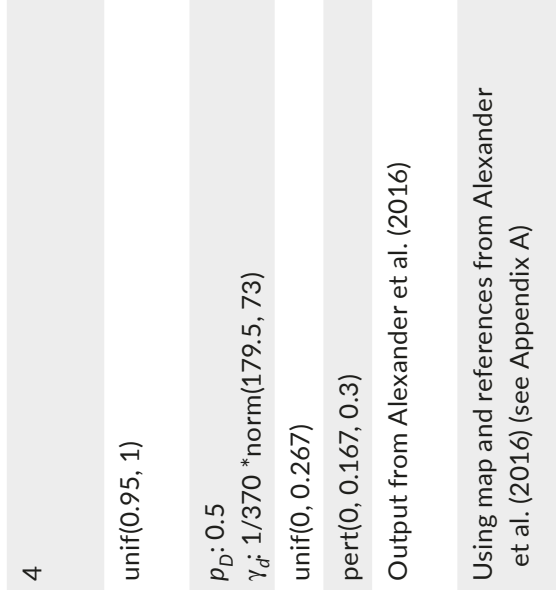

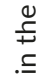

ఫु

嵌

品

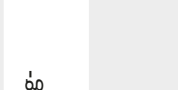

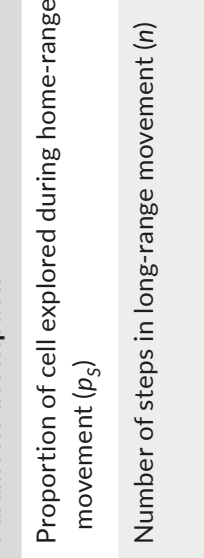

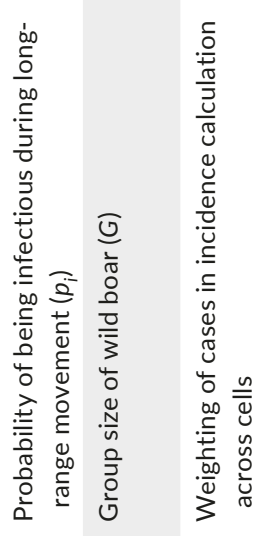

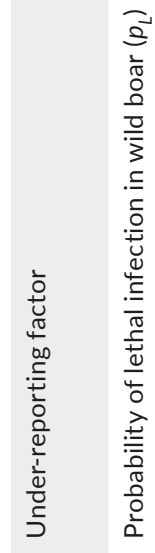

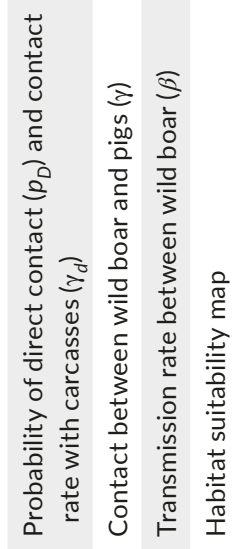


metrics indicate what a single simulation looks like, regarding severity and spread, and how much this varies across all the simulations. The third metric is a summary statistic indicating overall potential spread by stating the number of cells that have a case in at least one simulation (i.e. the size of the risk region).

\section{8 | Sensitivity analysis}

We perform a sensitivity analysis in order to assess the robustness of our results to uncertainty in parameter values. We use realistic alternative parameter values where possible (Table 2 ) and compare against the baseline results when using 2014 cases to predict the probability of infection in 2015 . To compare the sensitivity of the model to each of these uncertainties, we use the same three metrics to measure severity and spread of the outbreak as for the 10 control measures.

\section{3 | RESULTS}

We plot the spatial probability of at least one infection of ASF in 2015 due to our estimated incidence of ASF in 2014 in Figure 2. This is

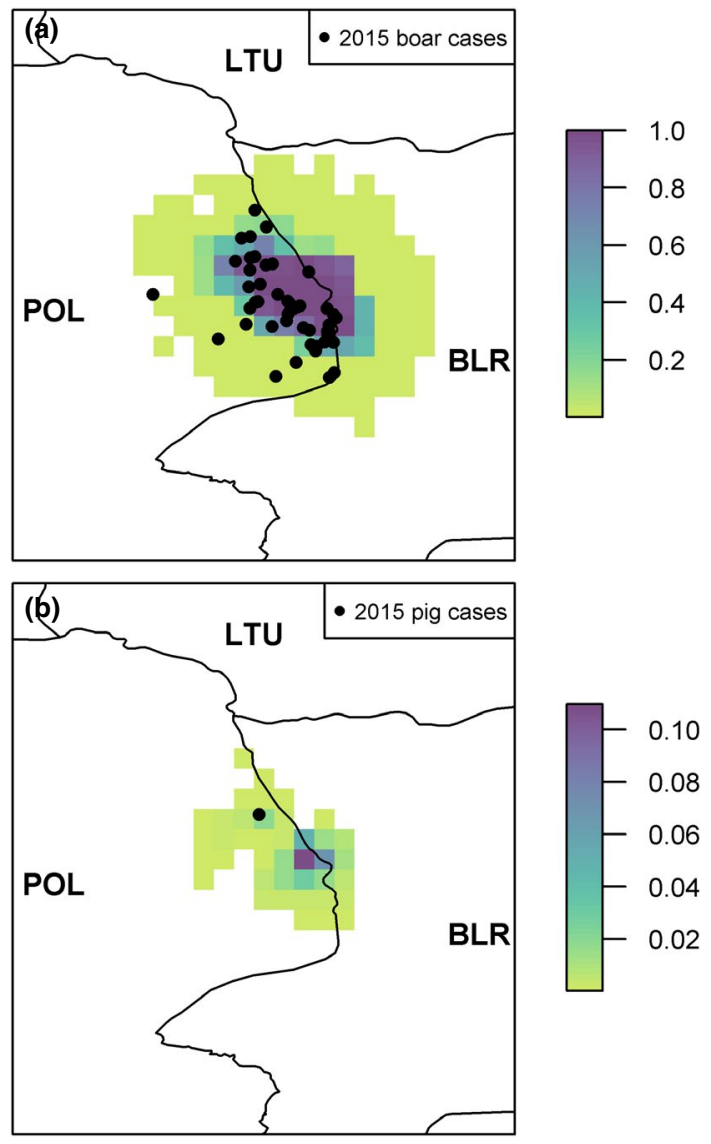

FIGURE 2 The probability of at least one infection with ASF virus in wild boar (a) and pigs (b) in 2015 caused by the movement of wild boar over one year. Black circles represent reported cases of ASF in wild boar and pigs in 2015. Countries are indicated by their ISO3 code assuming that one dispersal movement per wild boar can take place each year. The risk to wild boars is significantly higher than the risk to pigs, with the highest probability in any cell being 0.1 for pigs, that is a $10 \%$ chance that a new pig case would occur in that cell. The outer edges of the risk region have very low probabilities. As we are running 10,000 simulations only, it is possible that neighbouring cells to the edge could also have a non-zero probability of infection, which is $<1 \times 10^{-4}$. However, cells much further than one neighbour away from the risk region would have negligible risk according to our model due to our imposed limit for distance that boar can travel during dispersal.

Infection in wild boar could occur due to transmission from an infectious live wild boar or from contact with an infected carcass. The $2.5 \%, 50 \%$ and $97.5 \%$ quantiles of the number of new infections in wild boar are estimated separately for those caused by live wild boar contact or contact with carcasses (Figure 3). This highlights that infection in wild boar is more frequently caused by contact with live wild boar (see also Appendix C for the probability of at least one case in 2015 in wild boar split by live wild boar or carcasses). The upper percentiles for live wild boar indicate twice as many infections compared with those due to carcass transmission (Figure $3 c, f$ ), and this difference is even more noticeable for the lower and median percentiles. This is because the estimate for $R_{0}$ is lower on average for wild boar carcasses than contact with live wild boar since wild boar have a high contact rate with those in their social group, and can come into contact, albeit at a lower frequency, with those outside their social group. This outweighs the longer infectious period for wild boar carcasses. The upper percentile plots outline a smaller region of risk than the risk maps in Figure 2, indicating that in only a small percentage of simulations do we estimate that wild boar spread could be wider than this reduced region. In general, the number of cases that we predict is lower than the total number of wild boar cases that were reported (with an under-reporting factor included). This is reasonable since our model is only predicting those first initial cases that occur due to wild boar movement and does not include any secondary cases which could happen subsequently in that new area within the course of the year.

The midpoint of the 2015 cases was $7.204 \mathrm{~km}$ away from the midpoint of the 2014 cases, and all of the cases in 2015 were within $9.163 \mathrm{~km}$ of their nearest 2014 cases. Thus, the disease spread remained relatively confined in this year of the outbreak.

\subsection{Probability of new infections in 2016 given 2015 reported cases}

We now predict the probability of at least one new infection of ASF in wild boar and pigs in 2016 due to movement of wild boar (Figure 4). The probability of ASF in wild boar is on a similar scale to that predicted for 2015 , with up to $100 \%$ chance of ASF infection in some cells. In comparison, the probability of ASF infection in pigs is much lower in 2016 than in 2015 with a maximum $2 \%$ chance of transmission in a cell. However, in both cases the number of cells in 

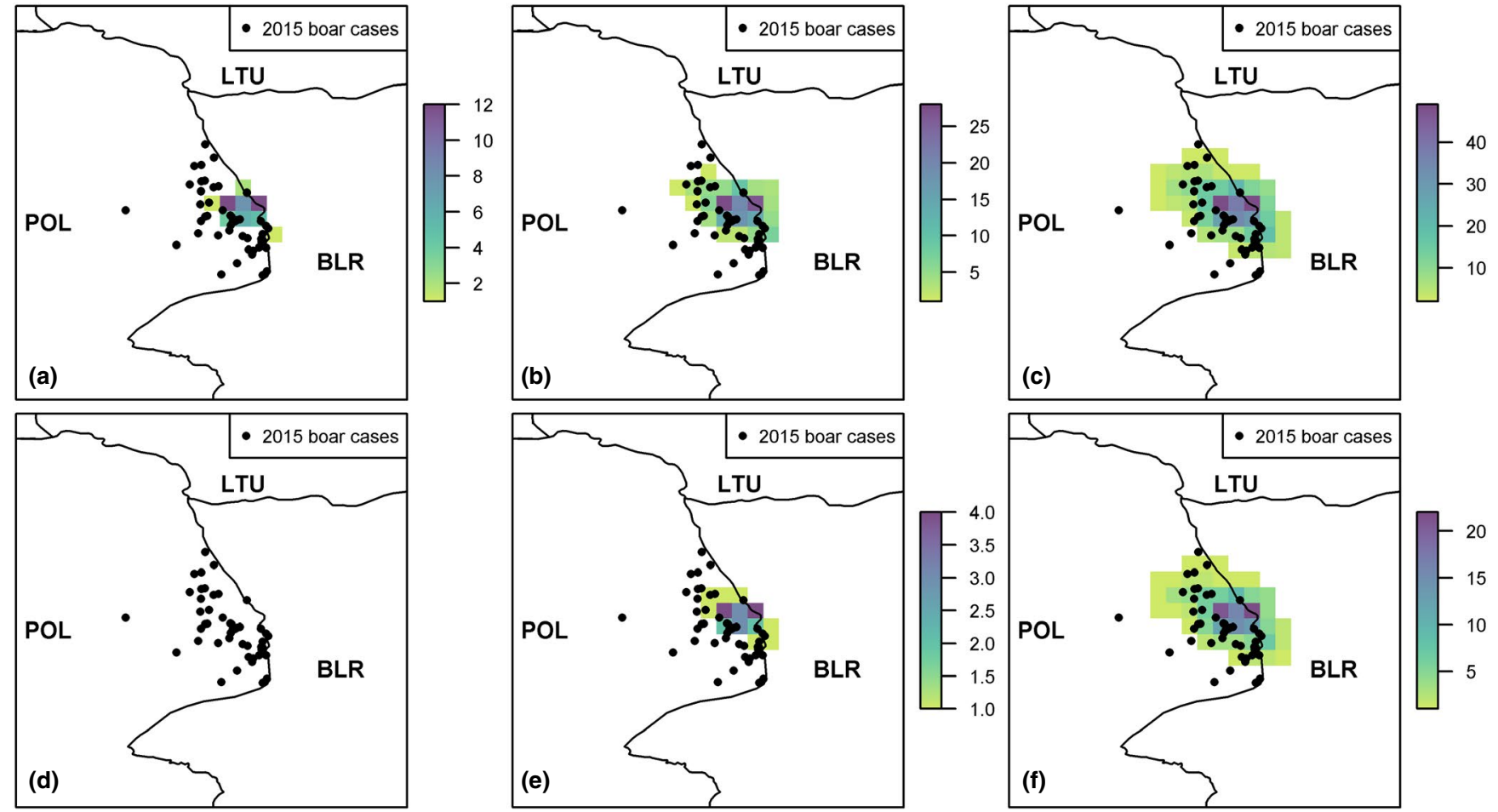

FIGURE 3 The $2.5 \%, 50 \%$ and $97.5 \%$ quantiles of the number of new infections with ASF virus in wild boar in 2015 due to transmission by live wild boar ( $a-c$, respectively) and by contact with wild boar carcasses ( $d-f$, respectively). Note the different scales on each subplot. Black circles indicate reported cases of ASF in wild boar in 2015. Countries are indicated by their ISO3 code

FIGURE 4 The probability of at least one new infection with ASF virus in wild boar and pigs in 2016 (a and b). In (c and d) the probability of at least one infection with ASF virus in wild boar is split between transmission due to infected live wild boar and by contact with infected wild boar carcasses, respectively. In (a, c and d) wild boar cases in 2016 are plotted, while in (b) pig cases in 2016 are plotted. Each of the cases in 2016 is plotted with different shapes to represent which cluster the cases belong to according to the cluster analysis. Countries are represented by their ISO3 code
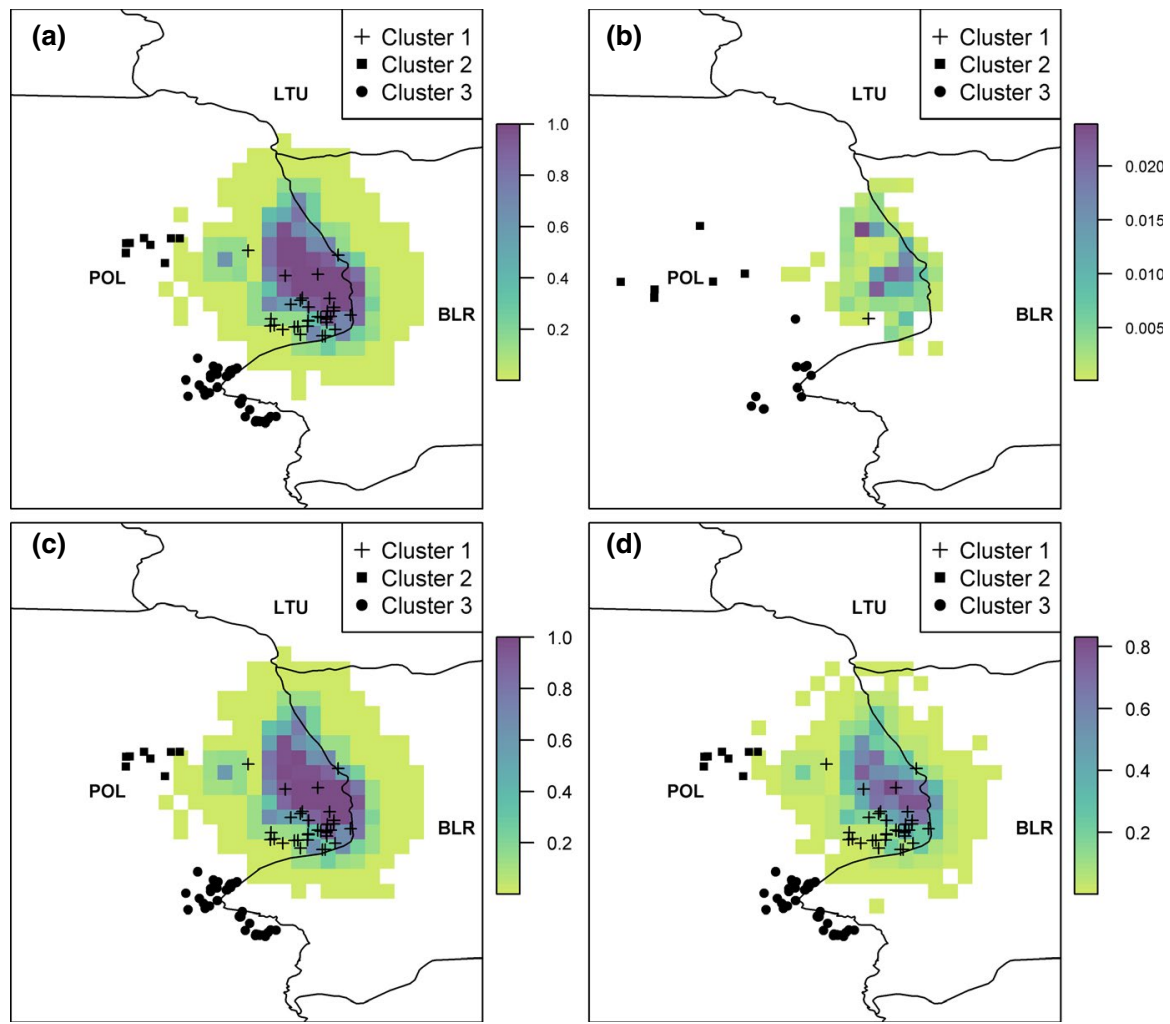

which a non-zero probability of transmission occurs is much higher due to the movement of wild boar further out from the original cases in 2014 after two dispersal events. In Figure 4c, d, we plot separately the probability of cases occurring due to wild boar movement and wild boar carcasses. As before, this indicates that under the current parameterization of the model, wild boar transmission by contact with other live wild boar is more frequent than contact with carcasses. 
A cluster analysis of the 2016 cases of ASF indicates that there are 3 distinct clusters of cases that year (see Appendix $C$ for methods and further results of the cluster analysis). This in itself suggests that the cases in the different clusters are unlikely to be caused by wild boar dispersion from the 2015 Poland cases as such spread would be expected to be more spatially homogenous. We indicate in Figure 4 which cluster all of the cases belong to. These are cluster 1 close to the cases in 2015 and then two separate clusters to the west (cluster 2) and to the south (cluster 3 ). The model indicates that it is highly unlikely (probability $\leq .0001$ ) that the cases of wild boar and pigs in the southerly or westerly clusters were due to wild boar movement from the 2015 Poland cases alone.

We plot the timing of cases of wild boar and pigs in 2016 reported throughout the year (Figure 5). Pig cases only occurred in 4 months of the year, whereas there were wild boar cases in nearly all 12 months. Figure 5 indicates that in both the southerly and the westerly clusters, the pig cases occurred first. For example, in the southerly cluster, most of the wild boar cases occurred in the 4th quarter of the year, while all of the pig cases occurred in August and September, the 3rd quarter. A similar story emerges in the westerly cluster. Our model predictions indicate that these pig cases would not have been caused by wild boar movement as the probability of infection in pigs from wild boar movement is very low (Figure 4).

\section{2 | Scenario analyses}

We compare the 10 control scenarios against the baseline scenario to assess their effectiveness using the severity and spread measures outlined above. We compute these metrics separately for wild boar (Figure 6). Full results of each of the scenarios are provided in Appendix D, for both wild boar and pigs.

With regard to reducing the total number of wild boar cases (Figure 6a), the hunting strategies appear to be the most successful, with $\mathrm{H} 4$, in which the wild boar population is reduced to $1 / 4$ of their original population size (in cells with cases in 2014) but $50 \%$ of wild boar undergo long-range dispersal, having the lowest number of cases per simulation. This strategy reduces the median number of cases per simulation by $75 \%$ from the baseline with $\mathrm{H} 2$ and $\mathrm{H} 3$ joint second at $63 \%$. Perhaps surprisingly, $\mathrm{H} 4$ is a more successful strategy in reducing the total number of wild boar cases than $\mathrm{H} 2$ in which wild boar are also reduced to $1 / 4$ of their original population size but only $25 \%$ of wild boar undergo long-range dispersal. The reason for this is that there are more wild boar dispersing, and therefore, there is a greater likelihood that the wild boar will move outside of the original non-zero incidence region. Normally, the area which had cases previously is the area where most cases will occur, due to either non-dispersing wild boar or wild boar dispersing to these cells as they are close by. Therefore, more wild boar dispersing leads to more wild boar leaving these cells. But there are many cells which the wild boar could move to, and hence, each cell outside the nonzero incidence area only receives a small number of wild boar and therefore the probability of a case occurring in each cell is low.

Similarly, the number of cells which have a wild boar case per simulation is lowest for $\mathrm{H} 4$ with reduction by $22 \%$ in the median number of cells infected per simulation. This is followed by H3, F1 and F2 (two scenarios with fences of $10 \mathrm{~km}$ width), all with a reduction of $15 \%$. However, this does not mean that only 27 cells could be infected in total (the median for the baseline), but in each simulation on average 27 cells have infection occur within them. As can be seen from Figure $6 \mathrm{c}$, the total number of cells that have a non-zero probability of infection is highest for $\mathrm{H} 3$, with 180 cells in the region at risk (an increase of $21 \%$ from the baseline). All of the hunting strategies lead to a bigger region of potential risk than the baseline scenario. This is due to the increase in the dispersal rate which leads to wild boar entering more cells further away from the original non-zero incidence area, potentially causing infection in those cells with a low probability.

In comparison, the fencing strategy $\mathrm{F} 3$, in which a $95 \%$ successful fence of $20 \mathrm{~km}$ width is built, is better for reducing the spread as the risk region is reduced by $9 \%$ from the baseline scenario to 131 cells. In the baseline scenario, nearly all of the cases occur within $20 \mathrm{~km}$ of the 2014 cases; thus, the addition of a $20 \mathrm{~km}$ fence does

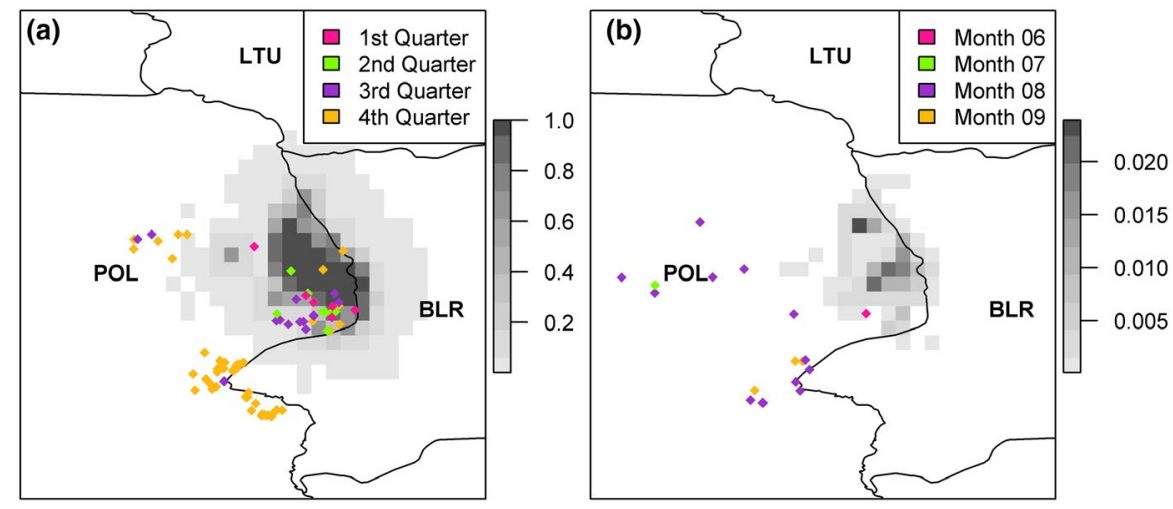

FIGURE 5 The probability of at least one infection with ASF virus in wild boar (a) and pigs (b) in 2016 is plotted in greyscale, while the reported cases of ASF in 2016 are plotted in colour depending on the time of year the case was reported. In (a), wild boar cases are plotted based on the quarter of the year they occur: 1st-Jan, Feb, Mar; 2nd-Apr, May, June; 3rd-July, Aug, Sept; and 4th-Oct, Nov, Dec. In (b), pig cases are plotted according to the month in 2016 they occur. Countries are represented by their ISO3 code 
(a)

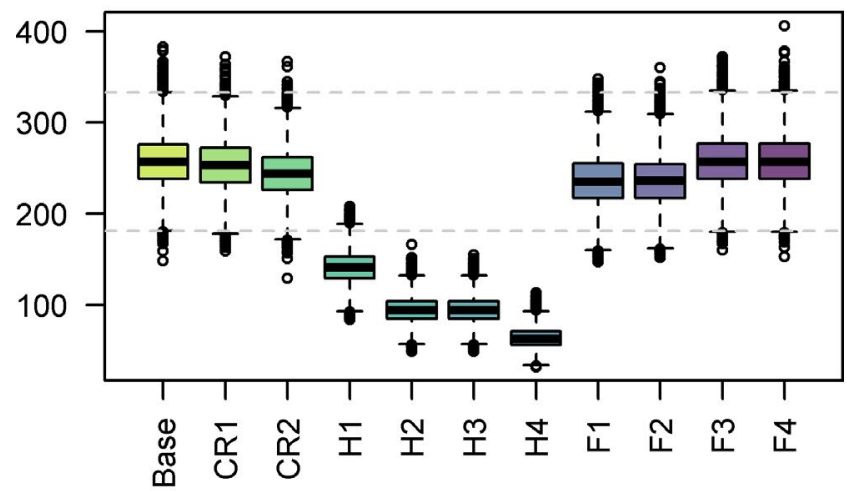

(b) Total Number of Cells with Cases per Simulation

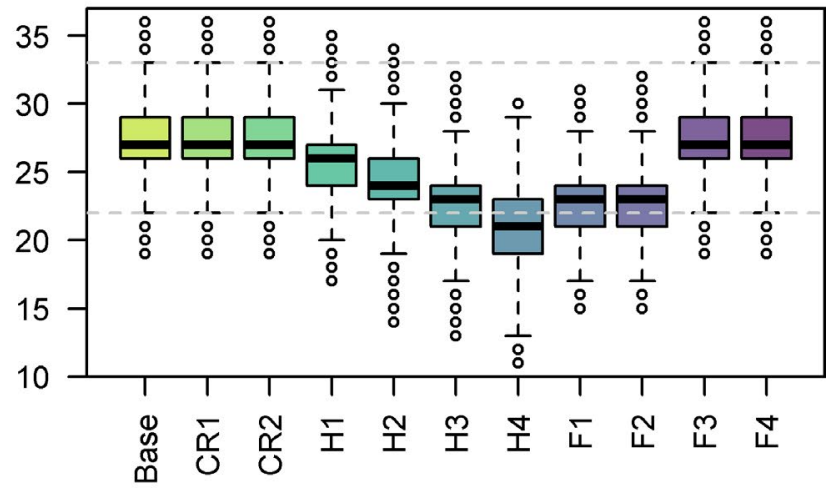

(c)

Total Number of Cells in Risk Region

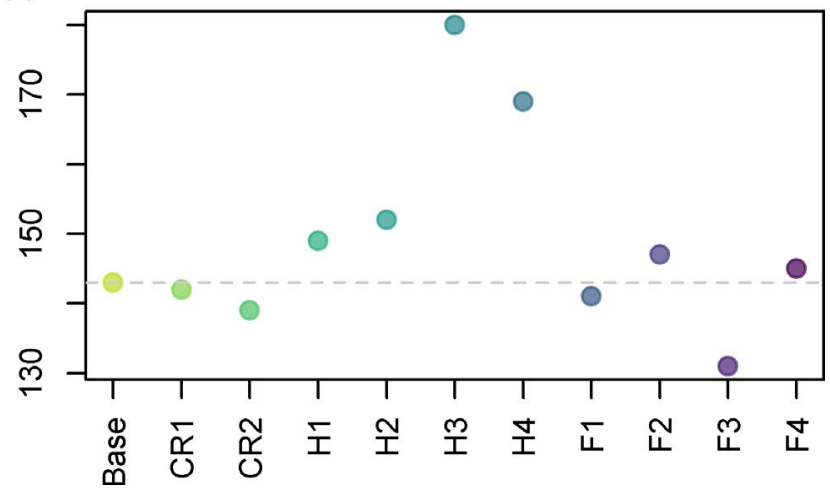

FIGURE 6 The baseline and scenario results are summarized using three metrics-the total number of new wild boar cases for each simulation (a); the total number of $100-\mathrm{km}^{2}$ cells which have at least one new wild boar case occurring for each simulation (b); and the total number of cells with at least one new case occurring over all simulations (i.e. the extent of the risk region, c). 'Base' in the plot refers to the baseline 2015 results, while each scenario is represented by a reference name as outlined in the Methods section, where 'CR' refers to the carcass removal scenarios, ' $\mathrm{H}$ ' to the hunting scenarios and ' $F$ ' to the fencing scenarios

not reduce the total number of cases, but it does reduce the possibility of infrequent cases occurring further away, provided the fence is effective. When the fence is not effective at $20 \mathrm{~km}$, scenario F4, the results are indistinguishable from the baseline scenario. On the other hand, the scenarios with a fence of width $10 \mathrm{~km}$ (F1, F2) reduce the number of cells with cases per simulation because the wild boar within the fence are confined to a smaller region. However, as the fence does not cover all the cells in which we have predicted non-zero incidence based on 2014 cases (as can be seen in Appendix $\mathrm{B})$, the size of the risk region does not change noticeably $(<3 \%)$, as there are infected wild boar outside the fence before the start of the simulation. However, there is a reduction in total number of cases under these scenarios, for a similar reason as above for hunting-the large number of cells to disperse to means fewer infected wild boar are entering each cell outside the fenced area, and therefore, there is less chance of a case occurring in each cell. Carcass removal (CR1, CR2) does not change the spread of the disease significantly as wild boar are still able to move the same amount, but it does reduce the chances of those dead wild boar infecting others and hence the total number of cases is reduced from the baseline, by $5 \%$ for CR2.

\section{3 | Sensitivity analysis}

The sensitivity of the model to the 17 different sensitivity analysis outlined in Table 2 is presented in Figure 7 for both wild boar and pigs. An alternative plot indicating the percentage change in the results for each sensitivity analysis compared to the baseline is included in Appendix $\mathrm{E}$. The model is not sensitive to many of these parameters within biologically realistic ranges, including parameters relating to wild boar ecology-group size (GSlow, GShigh) and contact with carcasses (CRC), ASF-related parameters-transmission rate (TR) and lethality of the disease (LI), and to habitat suitability (HS), which is created using potentially different data and methodology. Some of the parameters affect only one aspect of the results, for example PrevE, which smooths initial incidence more broadly than in the baseline model, leads to an increase in the number of cells with cases, although the third quartile is still lower than the upper whisker of the baseline boxplot (Figure 7b). Similarly, LRlow and LRhigh, which relate to the length of long-distance movement, affect the size of the risk region (Figure 7c) but do not affect the number of cells with cases per simulation (Figure 7b). In other words, on average, the number of cells expected to have cases remains the same but which specific cells those are change noticeably from one simulation to the next. We investigated whether this increase in the risk region affected our prediction of separate disease clusters in 2016 by applying the value of LRhigh in the 2015-2016 disease situation (results not shown). We found that the probability of at least one infection in wild boar was increased to 0.0001 for a number of cells in the southerly cluster, but for the westerly cluster the probability remained $<.0001$ for all cells with cases apart from one on the edge of the cluster.

The parameters that the model results are most sensitive to, for infection in wild boar, are the size of the home range (HRlow, HRhigh), no weighting of incidence (PrevN), the underlying wild boar abundance map (BA) and the under-reporting factor, as all three metrics are affected. As expected, increasing the home range (HRhigh) and the under-reporting factor (UFhigh) leads to increases in all three metrics. UFhigh has the biggest increase, but this value was an assumption because there were no data to estimate this parameter effectively. PrevN leads to a significant decrease in the 


\section{Total Number of Cases per Simulation}
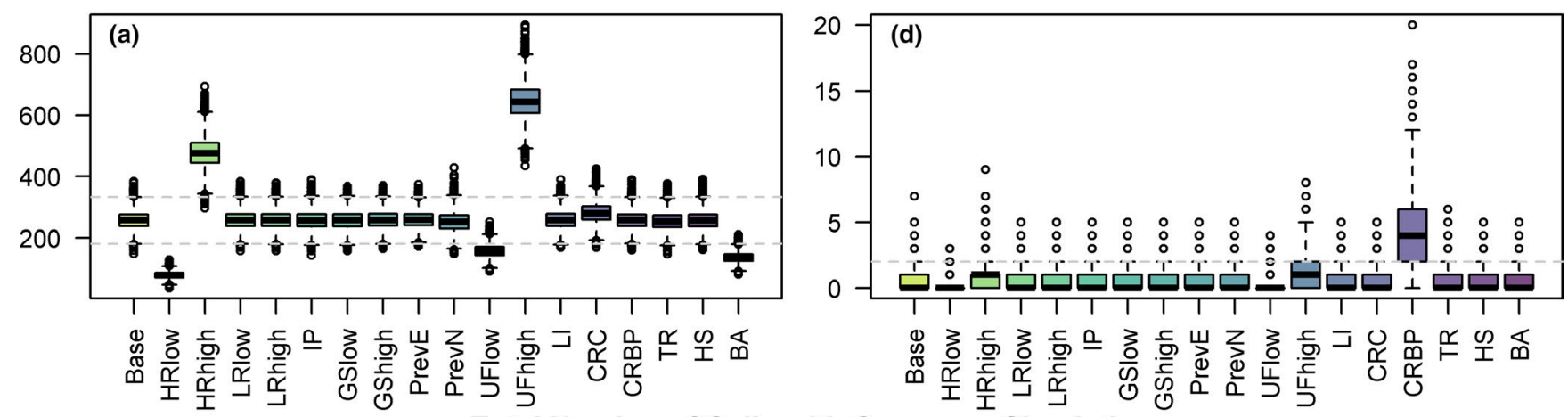

Total Number of Cells with Cases per Simulation

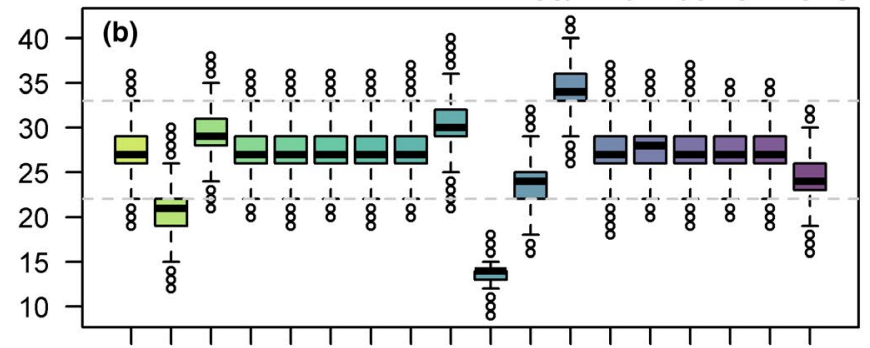

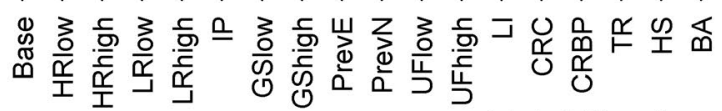

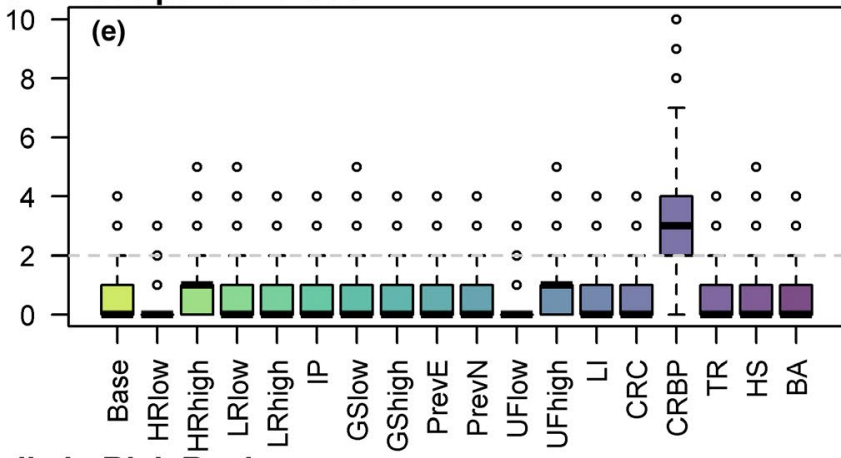

Total Number of Cells in Risk Region
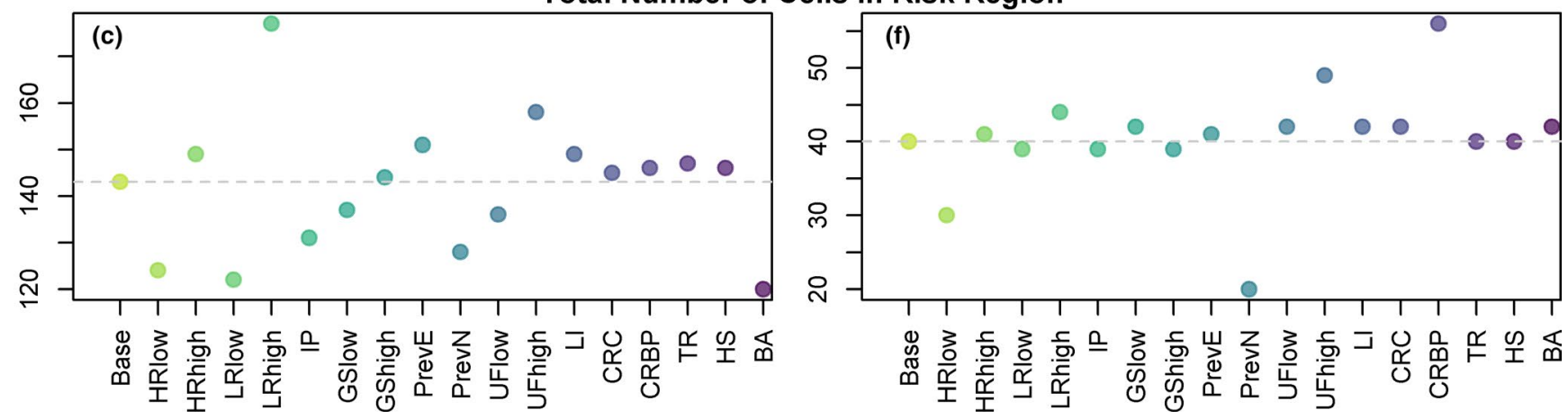

FIGURE 7 The baseline and 17 sensitivity analyses are summarized using three metrics-the total number of new cases per simulation (a and d); the total number of $100-\mathrm{km}^{2}$ cells which have at least one new case occurring for each simulation ( $b$ and e); and the total number of cells with at least one new case occurring over all simulations (c and f). In $(a-c)$ the results are for wild boar, and in ( $d-f$ ) for pigs. 'Base' in the plot refers to the baseline 2015 results, while each sensitivity analysis is represented by a reference name as outlined in Table 2

number of cells with cases per simulation (the median is reduced by $48 \%$ from the baseline median, Figure $7 \mathrm{~b}$ ) as the initial incidence is concentrated into fewer cells. Changing the wild boar abundance map (BA) reduces all three metrics because the estimates for wild boar abundance in Europe by Lewis et al. (2017) are lower than the original values based on Alexander et al. (2016). This has most impact on the size of the risk region, reducing it by $16 \%$. These parameters also affect the model results for infection in pigs (Figure $7 d-f$ ), and, in particular, PrevN results in a 50\% decrease for the size of the risk region (Figure $7 f$ ). The only parameter which significantly affects the metrics for the probability of infection in pigs, but does not affect probability of infection in wild boar, is CRBP, which is the contact rate between wild boar and pigs. This parameter increases the size of the risk region by $40 \%$ as well as increasing the mean number of cases per simulation ten-fold. Similar to UFhigh, the change to this parameter within the sensitivity analysis (increasing it ten-fold) was an assumption due to the paucity of data on this contact rate.

\section{4 | DISCUSSION}

We have investigated the role of natural wild boar movement in the transmission of ASF within Poland during 2014-2016. Our model suggests that the role of wild boar movement may be limited to only very local spread. Even when cases were within a relatively short distance from the cases the previous year, such as the southerly and westerly clusters of cases in 2016 , in none of our 10,000 simulations we did predict these cases due to wild boar movement. This is despite the fact that those clusters were 25 or $28 \mathrm{~km}$ away from the 2015 cases, respectively, and wild 
boar in the model have the potential to move $50 \mathrm{~km}$. In comparison, the cases from 2015 were much closer to the cases in 2014, with the maximum distance that a case in 2015 was from the nearest 2014 case of $9.163 \mathrm{~km}$. Similarly, the maximum distance that a case in cluster 1 in 2016 was from the nearest 2015 case is $16.889 \mathrm{~km}$. Overall, this indicates that on average, the spread of ASF has a moving boundary due to wild boar movement of $<25 \mathrm{~km}$ in a year. This is in line with statistical analysis of the cases in Europe, with estimates of spread at a rate of $2-5 \mathrm{~km} /$ month (Chenais et al., 2019) and $1.5 \mathrm{~km} /$ month (Podgórski \& Śmietanka, 2018). Our estimates, which are for spread by wild boar movement only, match the lower bounds of these statistical analyses. Overall, our findings regarding wild boar movement and ASF spread are in line with previous work, suggesting that wild boar movements have only a small role to play in mediumand long-distance spread of ASF (Podgórski \& Śmietanka, 2018) although this can depend on geographical location (Iglesias et al., 2018). However, our model predicts that transmission by live wild boar is more frequent than contact with carcasses in leading to new cases in ASF-free areas in wild boar, whereas other studies have suggested otherwise (Halasa, Boklund, Bøtner, Mortensen, \& Kjær, 2019; Lange et al., 2018; Pepin et al., 2020). This could be because the model is focused on spread rather than persistence of ASF in already infected areas. Our result is in line with the fact that studies on wild boar contact with carcasses suggest contact is primarily sniffing and poking rather than scavenging (Probst et al., 2017), and hence, wild boar would ingest lower viral doses. However, a recent study (Cukor et al., 2020) indicates that cannibalism in wild boar might be more prevalent than previous studies have found. If so, this could increase the transmission rate from wild boar carcasses and hence could reverse our finding that transmission by wild boar is more frequent than transmission by carcasses. Our result occurs in our model likely due to a higher contact rate with wild boar than with carcasses. However, we acknowledge that the exact ratio of live boar to carcass contacts is uncertain. In addition, while we included removal of ASF carcasses in the model, we did not consider the possibility that carcasses may only be removed after skeletonization has occurred (i.e. removing remaining bones). Similarly, transmission from a carcass is possible due to other wild animal scavengers (Probst et al., 2019). These would all increase the length of the transmission period or probability of transmission to live wild boar from carcasses, and hence potentially increase the impact of wild boar carcasses within the model.

The model suggests the westerly and southerly clusters of 2016 cases were not caused by wild boar movement from the 2015 Poland cases, and we corroborated this with the fact that cases in pig farms occurred first. Of course, it could be that cases were only found first in pigs and there was underlying infection in the wild boar population. However, as Poland was already aware of ASF in the country and was testing all wild boar carcasses found/hunted, it is unlikely that spread from the 2015 cases to these new clusters would have gone completely unnoticed, or that cases in the region between the clusters were not found, either before or after the pig cases. Therefore, we believe our model results reflect reality, and the conclusion has also been reached by the Polish authorities that the westerly cluster was caused first by human-mediated transmission to pigs and subsequent spillover to wild boar (pers. comm. General Veterinary Inspectorate) and supported by genetic tracking of ASF virus (Mazur-Panasiuk \& Woźniakowski, 2019). The main hypothesis for the original incursion in February 2014 in Poland is introduction from Belarus (Pejsak et al., 2014). The cases in 2016 in the southerly cluster are also very close to the Belarus border.

We have investigated the potential role that three control strategies, of varying effectiveness, could have on the transmission of the disease. Carcass removal overall had a positive effect on control of the disease, but the effect was minor. This follows from the model finding that live wild boar transmission was more important than transmission from carcasses. Hunting had the greatest potential to reduce the total number of cases but could also lead to the greatest region at risk. In contrast, fencing was successful in different ways depending on the width and the effectiveness of the fence. Fences which had a width of $10 \mathrm{~km}$ confined most of the wild boar to a small area, thus the number of cells with cases per simulation decreased, but it was possible for infected wild boar to be outside the fence when constructed and thus the size of the risk region was not decreased, as those wild boar could only move further away from the fence if dispersing. Thus, the fencing strategy of $20 \mathrm{~km}$ and $95 \%$ effectiveness was the most successful in reducing spread of the disease. However, it did not reduce the total number of cases as most cases occur within this $20 \mathrm{~km}$ area. If it is only possible to implement a single control strategy, the risk manager has to weigh up whether it is more important to reduce the region at risk of the virus or reduce the total number of boar that could become infected from the original cases. Given the potential for spread to go unnoticed in an area for some time, it would seem that reducing the region at risk would be deemed most important. Lange and co-authors (EFSA et al., 2017; Lange, 2015; Thulke \& Lange, 2017) have also considered the potential effects of control strategies separately, namely mass depopulation, a feeding ban and targeted hunting. These found that mass depopulation (provided it included prompt removal of carcasses) was the most successful strategy, but the levels required were not feasible under conventional wildlife management practices and, furthermore, that measures were more effective if implemented preventively rather than in an area with infected cases. In general, carcass removal has always been found to be beneficial for reducing cases (EFSA et al., 2018). However, as already stated, many of the recent countries with new incursions have implemented a combined strategy of 3 control strategies (fencing, hunting and carcass removal) in different zones. We did not combine intervention strategies, as our focus was on the movement model rather than the control strategies; however, Lange et al. (2018) considered various strategies combining zoning, hunting, carcass removal and fencing. Similar to our control strategy of fences with $10 \mathrm{~km}$ width, they found that fencing may be ineffective if based on locations of 
reported cases and not on locations of actual infected wild boar, but in general they found fencing to be a less successful strategy and instead focussed on combining intensive hunting with carcass removal. However, as also noted by Lange et al. (2018), further studies on wild boar ecology are required to understand how combined interventions may affect wild boar movement and group interactions, and therefore, combining interventions within models is subject to high uncertainty.

Our model relies on data on many different aspects of the ASF situation, such as the reporting of cases, movement ecology, matrilineal group and territorial dynamics, transmission of disease and contact with carcasses. Added to this is the fact that these are all applied to a wild rather than domestic species, and thus, undoubtedly there is less availability of or reliability in the data. For example, many of our parameters are determined by studies on pigs as there are few experiments determining transmission and survival rates for wild boar. As the model is stochastic, many of our parameters incorporate natural variation which reduces our uncertainty in our ability to cover likely values of each parameter, even if there could still be uncertainty in the shape of the distribution. Most of the distributions for parameters are pert or uniform to incorporate our lack of detailed knowledge. Due to the high computational requirements needed to perform the movement section of the model stochastically, this part of the model is deterministic (i.e. there are no distributions regarding the number of steps a wild boar will take or the percentage of wild boar undergoing dispersal). While it is likely that different wild boar could have a wide range of dispersal distances, we assume that infected wild boar would not be able to move very far, and thus, the lack of variation in this parameter should not be so important. Similarly, there is a great deal of uncertainty over the proportion of wild boar carcasses that are found and hence the under-reporting of wild boar cases. We found that the model was sensitive to this parameter, and hence, it is deserving of further investigation to determine a biologically sound upper bound. The model was more sensitive to the size of home-range movement than the length of long-distance dispersal of wild boar. While long-distance dispersal can affect the size of the risk region and hence which cells have infection present, it does not increase the chances of infection occurring in each cell. However, home-range movement directly affects the number of susceptible wild boar that each infected wild boar can contact, and hence, increasing the home range increases the number of new infections, and this is true whether the wild boar has undergone long-distance dispersal or not. Another parameter the model was sensitive to was the contact rate between wild boar and pigs, which was also highly uncertain. We found only one study which measured the number of direct contacts on a farm between wild boar and domestic pigs and, crucially, also stated the approximate number of wild boar in that area (Kukielka et al., 2013). However, this was on an open, free-ranging farm, which is not typical for Poland, and hence may overestimate the number of direct contacts. Uncertainty in this parameter could be reduced by further studies analysing the number of direct contacts between domestic pigs and wild boar, for example by using proximity loggers.
Wild boar abundance across Europe at $100 \mathrm{~km}^{2}$ was estimated by adapting the abundance map of Alexander et al. (2016). This is subject to high uncertainty due to the difficulties in extrapolating abundance across large areas when creating the original map, uncertainties and limitations in the original collection of abundance data, and in our method to convert to abundance values from scores. Furthermore, this is a static map whereas the population size and distribution of wild boar are constantly changing, especially considering the potential effects of ASF within a wild boar population, such as death, increased hunting and disturbance. When calculating the number of susceptible wild boar, we modified the abundance values by subtracting the number of infected boar that year, but we did not include potential reductions in wild boar populations due to other factors, such as hunting, seasonal variation and natural death. These changes in wild boar abundance could potentially lower the frequency of disease transmission by reducing the pool of susceptible individuals. We assessed the sensitivity of the model to the wild boar abundance map by aligning the abundance against output from Lewis et al. (2017). This addresses some of the uncertainty arising from the original method to compute abundance. We found that the model was sensitive to this change, although notably the results indicate more strongly the relatively local role that wild boar have to play in transmission, as spread and severity of transmission were reduced under this change. We also assessed the sensitivity of the Alexander et al. (2016) habitat suitability map by using a recently published alternative map (ENETWILD consortium, Smith, Acevedo, \& Vicente, 2019), and found that the model outputs were robust to this change. Further updates to wild boar abundance data and subsequent abundance maps would improve the validity of our results.

We modelled dispersal of wild boar assuming that it was driven by habitat suitability. However, other multi-level factors may be influential or more important in wild boar movement (Clobert, 2001; Kay et al., 2017), such as dispersing away from highly dense areas or conversely dispersing towards areas with other boar. Our model does not take account of these other factors, and hence, our results may be sensitive to this modelling assumption. Similarly, wild boar contact structures may be much more complicated than the within-group and between-group method we have used (Pepin \& VerCauteren, 2016). We did not separately model male and female wild boar, although there are differences in home-range size and propensity to disperse between the sexes (Podgórski et al., 2014). While it is possible to include further wild boar intricacies in the model, these are also subject to high uncertainty due to the level of data required to parameterize them effectively. By modelling wild boar ecology at a coarser level of detail, we are able to make generalizations across species. Hence, our model is applicable to modelling the spread of disease by any terrestrial wildlife species that may undergo dispersal for various reasons although, if adapting it to another species, it is advisable to ensure the dispersal model is appropriate for the species. A generic model that can be adapted quickly is crucial for responding to new and emerging diseases in a timely fashion to predict risk hotspots and spread of disease (Miller \& Pepin, 2019).

One aspect of the model which requires careful consideration is the role of secondary infections. Our model is only predicting 
the initial infections in new cells based on the estimated prevalence from reported cases. The model does not incorporate any other wild boar or pigs that could become infected due to the initial predicted wild boar cases. Therefore, we underestimate the total number of infected wild boar in an area. However, if the model is used as a tool to predict locations of ASF-infected wild boar rather than the exact number, an underestimation of the total number will not affect interpretation of model outputs. On the other hand, if all wild boar do not disperse around the same time each year, then it could be possible for an infected wild boar to disperse to a new cell, infect another wild boar in that cell, the new infected wild boar undergo its dispersal event after this (but still within the same year), and lead to infection in another wild boar or pig. This effectively stretches the size of the region at risk for one year and thus potentially indicates a faster wavefront of the disease spread. However, given the low probabilities involved in transmission, dispersal and movement distance, the increase to the risk region should be minimal as most cells outside of $20 \mathrm{~km}$ already have very low probability of infection $(<2.5 \%$, Figure 3$)$. Further, most young male dispersal does occur around the same time each year (Truvé \& Lemel, 2003). The role of secondary infections should not affect the success of the $20-\mathrm{km}$ fencing strategy as these secondary infections will also be restricted within the fenced area, although it may increase the number of infected wild boar within the fence. However, hunting disturbance could cause dispersal events to happen more frequently based upon when hunting occurs rather than the dispersal season (Thurfjell et al., 2013). Therefore, it is possible that we are underestimating the risk region in the hunting strategies due to secondary infections from multiple dispersal events of infected wild boar during a single year. Prompt carcass removal should reduce the number of secondary infections and therefore could be a more effective control strategy in a model with secondary infections included. One way to investigate the potential for increased spread by multiple dispersal events more rigorously would be to run our model on a shorter time scale than 1 year. Our model works for whatever time scale is most relevant, provided parameter rates are changed accordingly. For example, to run the model monthly, data on the percentage of wild boar dispersing each month would need to be supplied. This could include different timings for young male wild boar dispersal compared with adult male and female dispersal. However, it was not possible to do here due to the lack of data on when throughout the year dispersal events occur. ASF has shown seasonal peaks in incidence (Chenais et al., 2019) which could be due to seasonal peaks in wild boar dispersal. However, if it occurs for an independent reason then if the two seasonal inputs coincide, this could promote the spread of ASF further. Understanding the mechanisms behind the seasonality in ASF cases is required to model this aspect of ASF transmission.

In all of the results, the model predicts the greatest risk in those cells which previously had cases (even in scenarios with high wild boar dispersal rates). This outcome is mostly occurring due to home-range movement as this always remains within the cell of origin. In reality, wild boar home range could spread across multiple cells as wild boar are not restricted by our cell boundaries. Further, in the model wild boar may choose to disperse to cells quite close by and therefore could move into cells that already had cases. Perhaps, in reality, wild boar would be more directed in their movement away from their original location (Morelle et al., 2015). Another potential explanation is that the model underestimates the effect of the disease within the wild boar population and specifically on the rates of wild boar dispersal. It may be that the dispersal rate increased due to the presence of the disease or disturbance from human intervention, whether carcass removal or other control strategies. Lastly, it could be that there are wild boar becoming infected but are recovering from the disease, and are therefore not reported as cases since cases are predominantly found via wild boar carcasses rather than active testing of the wild boar population. However, this seems unlikely given the current knowledge of the highly virulent nature of the disease, even when wild boar and pigs are infected with low doses (Pietschmann et al., 2015). All hunted wild boar are also tested for ASF which should reduce this potential source of bias.

While we have investigated the role that wild boar movement has in local transmission of ASF, other transmission pathways can also be important, such as movement of pigs from farm to farm, contaminated cars, shoes or clothing, or transport/removal of boar carcasses if the necessary biosecurity is not followed. We have not assessed the risk of ASF in new areas in Poland due to these transmission pathways as the focus was on understanding the role of wild boar movement. However, this model framework for transmission of ASF by wild boar movement can now be combined with other pathways in order to compute the risk of infection of ASF on a broader scale through many routes. The fine spatial scale of the model favours results that can be used to target surveillance activities by highlighting potential areas of high risk of infection that have yet to report cases. For example, as seen in Figures 2 and 4, there was a high risk of cases in wild boar in Belarus on the border with Poland during both 2015 and 2016, and hence, the model could have been used to indicate locations best to test for ASF in Belarus. Although the model was shown using a case study for Poland in 2014-2016, it is applicable across the whole of Europe, provided data on wild boar abundance and habitat suitability is available. Furthermore, the model can be run prospectively, using up-to-date data on locations of reported cases. Combining the ability to run the model on real-time data and for the whole of Europe at a fine spatial scale, the model has the potential to aid in ASF hotspot identification, and consequently, in developing riskbased surveillance plans, a necessity if ASF will be successfully controlled across Europe and worldwide.

\section{ACKNOWLEDGEMENTS}

This project is part of COMPARE (Collaborative management platform for detection and analysis of (re-)emerging and foodborne outbreaks in Europe) which received funding from the European 
Union's Horizon 2020 research and innovation programme under grant agreement no. 643476. T. Podgórski was financially supported by the National Science Centre, Poland (grant number 2014/15/B/ NZ9/01933).

\section{CONFLICT OF INTEREST}

The authors declare that there is no conflict of interest.

\section{ETHICAL APPROVAL}

The authors confirm that the ethical policies of the journal, as noted on the journal's author guidelines page, have been adhered to. No ethical approval was required as this is a review article with no original research data.

\section{DATA AVAILABILITY STATEMENT}

Data sharing is not applicable to this article as no new data were created or analysed in this study.

\section{ORCID}

Rachel A. Taylor (iD https://orcid.org/0000-0002-2739-6944 Tomasz Podgórski iD https://orcid.org/0000-0001-6858-7838

Robin R. L. Simons (iD https://orcid.org/0000-0002-9202-0265

Paul Gale iD https://orcid.org/0000-0001-8142-9957

Louise A. Kelly iD https://orcid.org/0000-0002-2242-0781

\section{REFERENCES}

Adkin, A., Coburn, H., England, T., Hall, S., Hartnett, E., Marooney, C., .. Cox, T. (2004). Risk assessment for the illegal import of contaminated meat and meat products into Great Britain and the subsequent exposure of GB livestock (IIRA): Foot and mouth disease (FMD), classical swine fever (CSF), African swine fever (ASF), swine vesicular disease (SVD). New Haw: Veterinary Laboratories Agency.

Alexander, N. S., Massei, G., \& Wint, W. (2016). The European Distribution of Sus Scrofa. Model outputs from the project described within the poster-where are all the boars? An attempt to gain a continental perspective. Open Health Data, 4(1), p.e1.

Andrzejewski, R., \& Jezierski, W. (1978). Management of a wild boar population and its effects on commercial land. Acta Theriologica, 23(19), 309-339. https://doi.org/10.4098/AT.arch.78-23

Blome, S., Gabriel, C., Dietze, K., Breithaupt, A., \& Beer, M. (2012). High virulence of African swine fever virus caucasus isolate in European wild boars of all ages. Emerging Infectious Diseases, 18(4), 708

Bosch, J., Rodríguez, A., Iglesias, I., Muñoz, M., Jurado, C., SánchezVizcaíno, J., \& De la Torre, A. (2017). Update on the risk of introduction of African swine fever by wild boar into disease-free European Union Countries. Transboundary and Emerging Diseases, 64(5), 14241432. https://doi.org/10.1111/tbed.12527

Chenais, E., Depner, K., Guberti, V., Dietze, K., Viltrop, A., \& Ståhl, K. (2019). Epidemiological considerations on African swine fever in Europe 2014-2018. Porcine Health Management, 5(1), 6.

Clobert, J. (2001). Dispersal. Oxford, UK: Oxford University Press.

Costard, S., Mur, L., Lubroth, J., Sanchez-Vizcaino, J., \& Pfeiffer, D. (2013). Epidemiology of African swine fever virus. Virus Research, 173(1), 191-197. https://doi.org/10.1016/j.virusres.2012.10.030

Cukor, J., Linda, R., Václavek, P., Mahlerová, K., Šatrán, P., \& Havránek, F. (2020). Confirmed cannibalism in wild boar and its possible role in African swine fever transmission. Transboundary and Emerging Diseases. https://doi.org/10.1111/tbed.13468
Cwynar, P., Stojkov, J., \& Wlazlak, K. (2019). African swine fever status in Europe. Viruses, 11(4), 310. https://doi.org/10.3390/v11040310

D'Eath, R. B., \& Turner, S. P. (2009). The natural behaviour of the pig. The welfare of pigs (pp. 13-45). Dordrecht, the Netherlands: Springer.

De la Torre, A., Bosch, J., Iglesias, I., Muñoz, M., Mur, L., Martínez-López, B., ... Sánchez-Vizcaíno, J. M. (2015). Assessing the risk of African swine fever introduction into the European Union by wild boar. Transboundary and Emerging Diseases, 62(3), 272-279. https://doi. org/10.1111/tbed.12129

EFSA, (European Food Safety Authority), Boklund, A., Cay, B., Depner, K., Földi, Z., Guberti, V., ... Gortázar, C. (2018). Scientific report on the epidemiological analyses of African swine fever in the European Union (November 2017 until November 2018). EFSA Journal, 16(11), 5494. https://doi.org/10.2903/j.efsa.2018.5494

EFSA, (European Food Safety Authority), Depner, K., Gortazar, C., Guberti, V., Masiulis, M., More, S., ... Abrahantes, J. C. (2017). Epidemiological analyses of African swine fever in the Baltic States and Poland. EFSA Journal, 15(11), e05068. https://doi.org/10.2903/j. efsa.2017.5068

ENETWILD consortium, Croft, S., Smith, G., Acevedo, P., \& Vicente, J. (2019). Wild boar in focus: Initial model outputs of wild boar distribution based on occurrence data and identification of priority areas for data collection. EFSA Supporting Publications, 16(1), 1533E. https:// doi.org/10.2903/sp.efsa.2019.EN-1533

European Union (2002). Council Directive 2002/60/EC of 27 June 2002 laying down specific provisions for the control of African swine fever and amending Directive 92/119/EEC as regards Teschen disease and African swine fever (Text with EEA relevance) OJ L 192, 20.7.2002.

European Union (2018). SANTE/7113/2015 - Rev 10: Strategic Approach to the management of African Swine Fever for the EU.

FAO (2014). Gridded Livestock of the World (GLW). Retrieved May, 2017, from http://www.fao.org/ag/againfo/resources/en/glw/home.html

FAO (2019). ASF China situation update. Retrieved April, 2019, from http:// www.fao.org/ag/againfo/programmes/en/empres/ASF/2019/Situa tion_update_2019_03_29.html

Gabriel, C., Blome, S., Malogolovkin, A., Parilov, S., Kolbasov, D., Teifke, J. P., \& Beer, M. (2011). Characterization of African swine fever virus Caucasus isolate in European wild boars. Emerging Infectious Diseases, 17(12), 2342. https://doi.org/10.3201/eid1712.110430

Guinat, C., Gubbins, S., Vergne, T., Gonzales, J., Dixon, L., \& Pfeiffer, D. (2016). Experimental pig-to-pig transmission dynamics for African swine fever virus, Georgia 2007/1 strain. Epidemiology \& Infection, 144(1), 25-34. https://doi.org/10.1017/S0950268815000862

Guinat, C., Reis, A. L., Netherton, C. L., Goatley, L., Pfeiffer, D. U., \& Dixon, L. (2014). Dynamics of African swine fever virus shedding and excretion in domestic pigs infected by intramuscular inoculation and contact transmission. Veterinary Research, 45(1), 93.

Halasa, T., Boklund, A., Bøtner, A., Mortensen, S., \& Kjær, L. J. (2019). Simulation of transmission and persistence of African swine fever in wild boar in Denmark. Preventive Veterinary Medicine, 167, 68-79.

Iglesias, I., Montes, F., Martínez, M., Perez, A., Gogin, A., Kolbasov, D., \& de la Torre, A. (2018). Spatio-temporal kriging analysis to identify the role of wild boar in the spread of African swine fever in the Russian Federation. Spatial Statistics, 28, 226-235. https://doi.org/10.1016/j. spasta.2018.07.002.

Kay, S. L., Fischer, J. W., Monaghan, A. J., Beasley, J. C., Boughton, R., Campbell, T. A., ... Kilgo, J. C. (2017). Quantifying drivers of wild pig movement across multiple spatial and temporal scales. Movement Ecology, 5(1), 14.

Keuling, O., Lauterbach, K., Stier, N., \& Roth, M. (2010). Hunter feedback of individually marked wild boar Sus scrofa L.: Dispersal and efficiency of hunting in northeastern Germany. European Journal of Wildlife Research, 56(2), 159-167. https://doi.org/10.1007/s1034 4-009-0296-x 
Kukielka, E., Barasona, J. A., Cowie, C. E., Drewe, J., Gortazar, C., Cotarelo, I., \& Vicente, J. (2013). Spatial and temporal interactions between livestock and wildlife in South Central Spain assessed by camera traps. Preventive Veterinary Medicine, 112(3-4), 213-221.

Lange, M. (2015). Alternative control strategies against ASF in wild boar populations. EFSA Supporting Publications, 12(7), 843E.

Lange, M., Guberti, V., \& Thulke, H. H. (2018). Understanding ASF spread and emergency control concepts in wild boar populations using individual-based modelling and spatio-temporal surveillance data. EFSA Supporting Publications, 15(11), 1521E.

Leaper, R., Massei, G., Gorman, M., \& Aspinall, R. (1999). The feasibility of reintroducing wild boar (Sus scrofa) to Scotland. Mammal Review, 29(4), 239-258. https://doi.org/10.1046/j.1365-2907.1999.29402 39. $x$

Lewis, J. S., Farnsworth, M. L., Burdett, C. L., Theobald, D. M., Gray, M., \& Miller, R. S. (2017). Biotic and abiotic factors predicting the global distribution and population density of an invasive large mammal. Scientific Reports, 7, 44152.

Massei, G., Genov, P., Staines, B., \& Gorman, M. (1997). Factors influencing home range and activity of wild boar (Sus scrofa) in a Mediterranean coastal area. Journal of Zoology, 242(3), 411-423. https://doi.org/10.1111/j.1469-7998.1997.tb03845.x

Massei, G., Kindberg, J., Licoppe, A., Gačić, D., Šprem, N., Kamler, J., ... Náhlik, A. (2015). Wild boar populations up, numbers of hunters down? A review of trends and implications for Europe. Pest Management Science, 71(4), 492-500. https://doi.org/10.1002/ ps.3965

Mazur-Panasiuk, N., \& Woźniakowski, G. (2019). The unique genetic variation within the $\mathrm{O} 174 \mathrm{~L}$ gene of Polish strains of African swine fever virus facilitates tracking virus origin. Archives of Virology, 64(6), 1667-1672.

Mebus, C. A., House, C., Gonzalvo, F. R., Pineda, J. M., Tapiador, J., Pire, J. J., ... Sanchez-Vizcaino, J. M. (1993). Survival of foot-andmouth disease, African swine fever, and hog cholera viruses in Spanish serrano cured hams and Iberian cured hams, shoulders and loins. Food Microbiology, 10(2), 133-143. https://doi.org/10.1006/ fmic.1993.1014

Miller, R. S., \& Pepin, K. M. (2019). BOARD INVITED REVIEW: Prospects for improving management of animal disease introductions using disease-dynamic models. Journal of Animal Science, 97(6), 2291-2307. https://doi.org/10.1093/jas/skz125

Mlynar, P. (2018). Ministerial Conference on "Eradication of African swine fever (ASF) in the EU and the long-term management of wild boar populations". African swine fever in wild boar in the Czech Republic. Ministry of Agriculture of the Czech Republic.

Morelle, K., Podgórski, T., Prévot, C., Keuling, O., Lehaire, F., \& Lejeune, P. (2015). Towards understanding wild boar Sus scrofa movement: A synthetic movement ecology approach. Mammal Review, 45(1), 15-29.

Morley, R. (1993). A model for the assessment of the animal disease risks associated with the importation of animals and animal products. Revue Scientifique Et Technique-Office International Des Epizooties, 12, 1055.

OIE (2015). Guidelines for wildlife disease surveillance: An overview. https:// www.oie.int/fileadmin/Home/eng/Internationa_Standard_Setting/ docs/pdf/WGWildlife/OIE_Guidance_Wildlife_Surveillance_Feb20 15.pdf, World Organisation for Animal Health

OIE (2020). World Organisation for Animal Health (OIE): World Animal Health Information Database (WAHIS) Interface. Retrieved March, 2020, from http://www.oie.int/wahis_2/public/wahid.php/Wahid home/Home

Olesen, A., Lohse, L., Boklund, A., Halasa, T., Belsham, G., Rasmussen, T., \& Bøtner, A. (2018). Short time window for transmissibility of African swine fever virus from a contaminated environment. Transboundary and Emerging Diseases. https://doi.org/10.1111/ tbed.12837
PAFF Committee (2019). African swine fever in wild boar in Belgium, July 2019. Retrieved from https://ec.europa.eu/food/sites/food/files/ animals/docs/reg-com_ahw_20190708_asf_bel.pdf

Pejsak, Z., Truszczyński, M., Kozak, E., \& Markowska-Daniel, I. (2014). Epidemiological analysis of two first cases of African swine fever in wild boars in Poland. Medycyna Weterynaryjna, 70(6), 369-372.

Penrith, M. L., Bastos, A. D., Etter, E. M., \& Beltrán-Alcrudo, D. (2019). Epidemiology of African swine fever in Africa today: Sylvatic cycle versus socio-economic imperatives. Transboundary and Emerging Diseases, 66(2), 672-686. https://doi.org/10.1111/tbed.13117

Pepin, K. M., Golnar, A., Abdo, Z., \& Podgorski, T. (2020). Ecological drivers of African swine fever virus persistence in wild boar populations: Insight for control. Ecology and Evolution, 10(6), 2846-2859. https:// doi.org/10.1002/ece3.6100

Pepin, K. M., \& VerCauteren, K. C. (2016). Disease-emergence dynamics and control in a socially-structured wildlife species. Scientific Reports, 6, 25150. https://doi.org/10.1038/srep25150

Pietschmann, J., Guinat, C., Beer, M., Pronin, V., Tauscher, K., Petrov, A., ... Blome, S. (2015). Course and transmission characteristics of oral low-dose infection of domestic pigs and European wild boar with a Caucasian African swine fever virus isolate. Archives of Virology, 160(7), 1657-1667. https://doi.org/10.1007/s00705-015-2430-2

Podgórski, T., Apollonio, M., \& Keuling, O. (2018). Contact rates in wild boar populations: Implications for disease transmission. The Journal of Wildlife Management, 82(6), 1210-1218. https://doi.org/10.1002/ jwmg. 21480

Podgórski, T., Scandura, M., \& Jędrzejewska, B. (2014). Next of kin next door-philopatry and socio-genetic population structure in wild boar. Journal of Zoology, 294(3), 190-197. https://doi.org/10.1111/ jzo.12167

Podgórski, T., \& Smietanka, K. (2018). Do wild boar movements drive the spread of African Swine Fever? Transboundary and Emerging Diseases, 65(6), 1588-1596.

Podgórski, T., \& Śmietanka, K. (2018). Do wild boar movements drive the spread of African Swine Fever? Transboundary and Emerging Diseases, 65(6), 1588.-1596.

Probst, C., Gethmann, J., Amler, S., Globig, A., Knoll, B., \& Conraths, F. J. (2019). The potential role of scavengers in spreading African swine fever among wild boar. Scientific Reports, 9(1), 1-13. https://doi. org/10.1038/s41598-019-47623-5

Probst, C., Globig, A., Knoll, B., Conraths, F. J., \& Depner, K. (2017). Behaviour of free ranging wild boar towards their dead fellows: Potential implications for the transmission of African swine fever. Royal Society Open Science, 4(5), 170054.

Sáaez-Royuela, C., \& Telleriia, J. (1986). The increased population of the wild boar (Sus scrofa L.) in Europe. Mammal Review, 16(2), 97-101. https://doi.org/10.1111/j.1365-2907.1986.tb00027.x

Sánchez-Cordón, P. J., Montoya, M., Reis, A. L., \& Dixon, L. K. (2018). African swine fever: A re-emerging viral disease threatening the global pig industry. The Veterinary Journal. https://doi.org/10.1016/j. tvjl.2017.12.025

Selva, N., Jędrzejewska, B., Jędrzejewski, W., \& Wajrak, A. (2005). Factors affecting carcass use by a guild of scavengers in European temperate woodland. Canadian Journal of Zoology, 83(12), 1590-1601. https:// doi.org/10.1139/z05-158

Simons, R. R. L., Horigan, V., Ip, S., Taylor, R. A., Crescio, M. I., Maurella, C., ... Adkin, A. (2019). A spatial risk assessment model framework for incursion of exotic animal disease into the European Union Member States. Microbial Risk Analysis, 13, 100075. https://doi.org/10.1016/j. mran.2019.05.001

Taylor, R. A., Berriman, A. D., Gale, P., Kelly, L. A., \& Snary, E. L. (2019). A generic framework for spatial quantitative risk assessments of infectious diseases: Lumpy skin disease case study. Transboundary and Emerging Diseases, 66(1), 131-143. https://doi.org/10.1111/ tbed.12993 
Thulke, H. H., \& Lange, M. (2017). Simulation-based investigation of ASF spread and control in wildlife without consideration of human non-compliance to biosecurity. EFSA Supporting Publications, 14(11), 1312E.

Thurfjell, H., Spong, G., \& Ericsson, G. (2013). Effects of hunting on wild boar Sus scrofa behaviour. Wildlife Biology, 19(1), 87-94.

Truvé, J., \& Lemel, J. (2003). Timing and distance of natal dispersal for wild boar Sus scrofa in Sweden. Wildlife Biology, 9(SUPPL 1), 51-57.

\section{SUPPORTING INFORMATION}

Additional supporting information may be found online in the Supporting Information section.
How to cite this article: Taylor RA, Podgórski T, Simons RRL, et al. Predicting spread and effective control measures for African swine fever-Should we blame the boars?. Transbound Emerg Dis. 2020;00:1-20. https://doi.org/10.1111/ tbed.13690 